\title{
Acoustic Properties of the Globular Photonic Crystals
}

\author{
N. F. Bunkin ${ }^{1}$ and V. S. Gorelik ${ }^{2}$ \\ ${ }^{1}$ A.M.Prokhorov General Physics Institute, Russian Academy of Sciences, \\ ${ }^{2}$ Lebedev Physical Institute, Russian Academy of Sciences, \\ Moscow, \\ Russia
}

\section{Introduction}

Modern technologies allow us to construct new nanomaterials with a periodic superstructure. In particular, the increasing interest has been recently shown in the so-called photonic (PTC) [1 - 4] and phononic (PNC) [5] crystals. In a case of PTC its structure is characterized by the refractive index, which periodically varies in space; the spatial period essentially exceeds the atomic sizes. PNCs are characterized by spatial periodic modulations of the sound velocity caused by the presence of the periodically settled elements of various materials (metals, polymers etc.) inside the sample. It is worth noting that PTC can at the same time be treated as a version of PNC.

PTCs and PNCs can be realized as one, two and three-dimensional structures. Among a wide variety of PTCs the special attention of researchers is paid to the crystal structures, whose lattice period is comparable with a wavelength of electromagnetic wave in the visible range. The periodicity of such PTC-structure results in presence of the so-called band-gaps located in the visible spectral range, i.e. in the spectral areas, where the electromagnetic waves can penetrate inside the sample only to a near-surface region with thickness of about a wavelength of light (0.4-0.8 microns). If the frequency of an electromagnetic wave is close to the band-gap edge, the group velocity of an electromagnetic wave drastically decreases, which results in a sharp increase of spectral density of electromagnetic radiation [2]. The numerous works are devoted to study of such effect for the visible range [2 - 4]. Other interesting feature of PTC consists in the existence of spectral bands characterized by a negative value of the effective refractive index, when the directions of phase and group velocities of an electromagnetic wave appear to be opposite to one another. At last we shall note that effective rest mass of photons in PTC is non-zero and can accept both positive and negative values. The absolute value of the effective rest mass of the photons in PTC is equal to $m=\frac{\hbar \omega}{c^{2}} \approx 10^{-36} \mathrm{~kg}$.

The properties of acoustic waves in PNC are in many respects similar to the properties of electromagnetic waves in PTC. In the given work the review of characteristic properties of acoustic waves in PNC in comparison with the corresponding properties of electromagnetic waves in PTC is given. In particular, the problems of finding the form of dispersion dependences $\omega(k)$ for acoustic waves together with the dispersion dependences of their 
group velocities and effective mass of the corresponding acoustic phonons are solved. The results of the theoretical analysis and the data of experimental studies of the optical and acoustic phenomena in PTC and PNC, including the studies of spectra of non-elastic scattering of light together with the experiments to observe the stimulated light scattering accompanying by the coherent oscillations of globules are reported.

\subsection{Theory of dispersion of electromagnetic and acoustic waves in one-dimensional PTC/PNC}

The one-dimensional dielectric medium with two alternating layers (see. Fig. 1) can be considered as a one-dimensional PTC. At the same time, such medium can either be regarded as a one-dimensional PNC characterized by specified propagation velocities of acoustic waves in each of layers. At the first stage, let us consider the dispersion law for electromagnetic waves on the basis of the theory developed earlier [5 - 7]. According to the technique described in detail in Ref. [5], in order to obtain the dispersion relation, we used the plane monochromatic wave approximation with allowance for the boundary conditions at the edges of the layers (see Fig. 1).

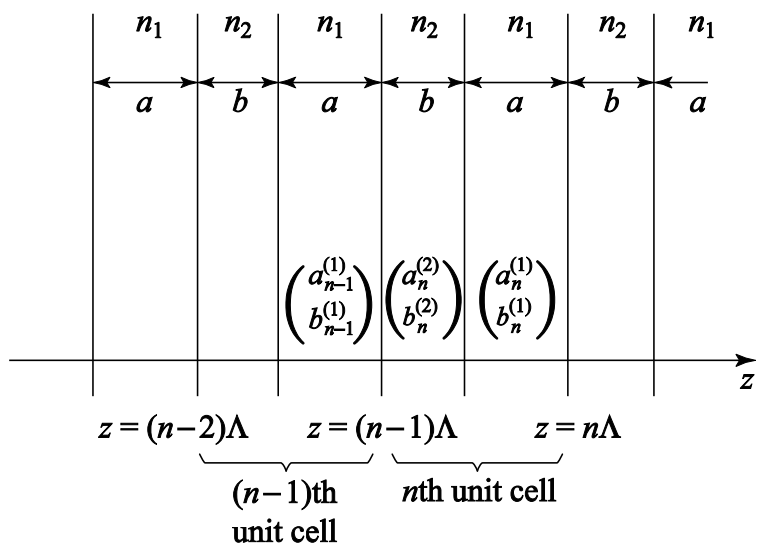

Fig. 1. Schematic of periodic layered medium and plane wave amplitudes corresponding to the $n$-th unit cell and its neighboring layers [5]

The periodic layered medium under study consists of two various substances with the following structure of the refractive index:

$$
n(z)= \begin{cases}n_{2}, & 0<z<b, \\ n_{1}, & b<z<\Lambda\end{cases}
$$

With making allowance for the periodicity of the refractive index, we arrive at:

$$
n(z)=n(z+\Lambda)
$$

Here the $z$-axis is perpendicular to the boundaries of layers, while $\Lambda$ is the spatial period of the superstructure. The general solution to the wave equation for the electric field vector can be sought for in the form 


$$
\mathbf{E}(\mathbf{r}, t)=\mathbf{E}_{0}(z) \exp \left[i\left(\omega t-k_{y} y\right)\right] .
$$

Here it is assumed that the wave propagates in $(y z)$ plane, whereas $k_{y}$ is the vector component that remains constant during the propagation through the medium. The electric field strength within each homogeneous layer can be represented as a sum of the incident and reflected plane waves. Complex amplitudes of these two waves are components of the column vector. Thus, the electric field in $\alpha$-th layer $(\alpha=1,2)$ of the $n$-th unit cell (see. Fig. 1) can be written in the form of the column vector

$$
\left(\begin{array}{c}
a_{n}^{(\alpha)} \\
b_{n}^{(\alpha)}
\end{array}\right), \alpha=1,2 .
$$

The distribution of the electric field strength in the layer under consideration can be represented as

$$
E(y, z)=\left\{a_{n}^{(\alpha)} \exp \left[-i k_{\alpha z}(z-n \Lambda)\right]+b_{n}^{(\alpha)} \exp \left[-i k_{\alpha z}(z-n \Lambda)\right]\right\} \exp \left(-i k_{y} y\right)
$$

where

$$
k_{\alpha z}=\sqrt{\left(\frac{n_{\alpha} \omega}{c}\right)^{2}-k_{y}^{2}}, \alpha=1,2
$$

The column vectors are related to each other by the conditions of continuity at the interfaces. As a consequence, only one vector (or two components of different vectors) can be chosen arbitrarily. For TE-waves (vector $E$ is perpendicular to the $y z$ plane), the condition for the continuity of the components $E_{x}$ and $H_{y}\left(H_{y} \sim \partial E_{x} / \partial z\right)[6,7]$ at the interfaces $z=(n-1) \Lambda$ and $z$ $=(n-1) \Lambda+b$ (see Fig. 1$)$ leads to the following equations:

$$
\begin{aligned}
& a_{n-1}+b_{n-1}=e^{i k_{2 z} \Lambda} c_{n}+e^{-i k_{2 z} \Lambda} d_{n}, i k_{1 z}\left(a_{n-1}-b_{n-1}\right)=i k_{2 z}\left(e^{i k_{2 z} \Lambda} c_{n}-e^{-i k_{2 z} \Lambda} d_{n}\right), \\
& e^{i k_{2 z} a} c_{n}+e^{-i k_{2 z} a} d_{n}=e^{i k_{1 z} a} a_{n}+e^{-i k_{1 z} a} b_{n}, i k_{2 z}\left(e^{i k_{2 z} a} c_{n}+e^{-i k_{2 z} a} d_{n}\right)=i k_{1 z}\left(e^{i k_{1 z} a} a_{n}+e^{-i k_{1 z} a} b_{n}\right) .
\end{aligned}
$$

These four equations can be written as a system of two matrix equations:

$$
\begin{gathered}
\left(\begin{array}{cc}
1 & 1 \\
1 & -1
\end{array}\right) \cdot\left(\begin{array}{l}
a_{n-1} \\
b_{n-1}
\end{array}\right)=\left(\begin{array}{cc}
\exp \left(i k_{2 z} \Lambda\right) & \exp \left(-i k_{2 z} \Lambda\right) \\
\frac{k_{2 z}}{k_{1 z}} \exp \left(i k_{2 z} \Lambda\right) & -\frac{k_{2 z}}{k_{1 z}} \exp \left(-i k_{2 z} \Lambda\right)
\end{array}\right) \cdot\left(\begin{array}{c}
c_{n} \\
d_{n}
\end{array}\right), \\
\left(\begin{array}{cc}
\exp \left(i k_{2 z} a\right) & \exp \left(-i k_{2 z} a\right) \\
\exp \left(i k_{2 z} a\right) & -\exp \left(-i k_{2 z} a\right)
\end{array}\right) \cdot\left(\begin{array}{l}
c_{n} \\
d_{n}
\end{array}\right)=\left(\begin{array}{cc}
\exp \left(i k_{1 z} a\right) & \exp \left(-i k_{1 z} a\right) \\
\frac{k_{1 z}}{k_{2 z}} \exp \left(i k_{1 z} a\right) & -\frac{k_{1 z}}{k_{2 z}} \exp \left(-i k_{1 z} a\right)
\end{array}\right) \cdot\left(\begin{array}{l}
a_{n} \\
b_{n}
\end{array}\right),
\end{gathered}
$$

where 


$$
a_{n} \equiv a_{n}^{(1)}, \quad b_{n} \equiv b_{n}^{(1)}, \quad c_{n} \equiv a_{n}^{(2)}, d_{n} \equiv b_{n}^{(2)} .
$$

Eliminating the column vector $\left(c_{n}, d_{n}\right)^{\mathrm{T}}$ from this system, we obtain the matrix equation

$$
\left(\begin{array}{l}
a_{n-1} \\
b_{n-1}
\end{array}\right)=\left(\begin{array}{ll}
A & B \\
C & D
\end{array}\right)\left(\begin{array}{l}
a_{n} \\
b_{n}
\end{array}\right) .
$$

The matrix elements in this equation are:

$$
\begin{aligned}
& A=\exp \left(i k_{1 z} a\right) \cdot\left[\cos k_{2 z} b+\frac{1}{2} i\left(\frac{k_{2 z}}{k_{1 z}}+\frac{k_{1 z}}{k_{2 z}}\right) \sin k_{2 z} b\right], B=\exp \left(-i k_{1 z} a\right) \cdot\left[\frac{1}{2} i\left(\frac{k_{2 z}}{k_{1 z}}-\frac{k_{1 z}}{k_{2 z}}\right) \sin k_{2 z} b\right] \\
& C=\exp \left(i k_{1 z} a\right) \cdot\left[-\frac{1}{2} i\left(\frac{k_{2 z}}{k_{1 z}}-\frac{k_{1 z}}{k_{2 z}}\right) \sin k_{2 z} b\right], D=\exp \left(-i k_{1 z} a\right) \cdot\left[\cos k_{2 z} b-\frac{1}{2} i\left(\frac{k_{2 z}}{k_{1 z}}+\frac{k_{1 z}}{k_{2 z}}\right) \sin k_{2 z} b\right] .
\end{aligned}
$$

Since the matrix (11) relates amplitudes of the field of two equivalent layers with identical refractive indices, it is unimodular, i.e.,

$$
\mathrm{AD}-\mathrm{BC}=1
$$

As was pointed out above, only one column vector is independent. For this vector one can choose, for instance, the column vector for layer 1 in the zero unit cell. The remaining column vectors of the equivalent layers are connected with the vector for the zero unit cell by the relation

$$
\left(\begin{array}{l}
a_{0} \\
b_{0}
\end{array}\right)=\left(\begin{array}{ll}
A & B \\
C & D
\end{array}\right)^{n}\left(\begin{array}{l}
a_{n} \\
b_{n}
\end{array}\right) .
$$

It follows from here that

$$
\left(\begin{array}{l}
a_{n} \\
b_{n}
\end{array}\right)=\left(\begin{array}{ll}
A & B \\
C & D
\end{array}\right)^{-n}\left(\begin{array}{l}
a_{0} \\
b_{0}
\end{array}\right),
$$

or, in view of (14)

$$
\left(\begin{array}{l}
a_{n} \\
b_{n}
\end{array}\right)=\left(\begin{array}{cc}
D & -B \\
-C & A
\end{array}\right)^{n}\left(\begin{array}{l}
a_{0} \\
b_{0}
\end{array}\right) .
$$

A periodic layered medium is equivalent to a one-dimensional PTC that is invariant under translations to the lattice constant. The lattice translation operator $T$ is defined by the expression

$$
T z=z-l \Lambda, l \in Z
$$

Thus, we arrive at

$$
\mathrm{TE}(z)=\mathbf{E}\left(T^{-1} z\right)=\mathbf{E}(z+l \Lambda)
$$


According to the Bloch theorem [6, 7], the vector of the electric field of the normal mode in the layered periodic medium has the form:

$$
\mathbf{E}=\mathbf{E}_{K}(z) \exp (-i K z) \exp \left[i\left(\omega t-k_{y} y\right)\right]
$$

where $\mathbf{E}_{K}(z)$ is the periodic function with the period $\Lambda$, i.e.,

$$
\mathbf{E}_{K}(z)=\mathbf{E}_{K}(z+\Lambda) \text {. }
$$

Using the column vector representation and expression (5), the periodicity condition (19) for the Bloch wave can be written as:

$$
\left(\begin{array}{l}
a_{n} \\
b_{n}
\end{array}\right)=\exp (-i K \Lambda)\left(\begin{array}{l}
a_{n-1} \\
b_{n-1}
\end{array}\right) .
$$

As follows from Eqns. (11) and (20), the column vector of the Bloch wave obeys the eigenvalue equation:

$$
\left(\begin{array}{ll}
A & B \\
C & D
\end{array}\right)\left(\begin{array}{l}
a_{n} \\
b_{n}
\end{array}\right)=\exp (i K \Lambda)\left(\begin{array}{l}
a_{n} \\
b_{n}
\end{array}\right) .
$$

Thus, the phase factor is the eigenvalue of the translation matrix $(A B C D)$ and satisfies the characteristic equation

$$
\operatorname{det}\left(\begin{array}{cc}
A-\exp (i K \Lambda) & B \\
C & D-\exp (i K \Lambda)
\end{array}\right)=0 .
$$

The solution to this equation has the form

$$
\exp (i K \Lambda)=\frac{1}{2}(A+D) \pm \sqrt{\frac{1}{2}(A+D)^{2}-1}
$$

Eigenvectors corresponding to these eigenvalues are solutions to Eqn. (21), and accurate to an arbitrary constant they can be represented in the form

$$
\left(\begin{array}{l}
a_{0} \\
b_{0}
\end{array}\right)=\left(\begin{array}{c}
B \\
\exp (i K \Lambda)-A
\end{array}\right)
$$

According to (20), the corresponding column eigenvector for the $n$-th unit cell is

$$
\left(\begin{array}{l}
a_{n} \\
b_{n}
\end{array}\right)=\exp (-i n K \Lambda)\left(\begin{array}{c}
B \\
\exp (i K \Lambda)-A
\end{array}\right) .
$$

The Bloch waves obtained from (23) and (24) can be considered as eigenvectors of the translation matrix with the eigenvalues $\exp (i K \Lambda)$, given by Eqn. (22); this equation results in the dispersion relation of the kind: 


$$
K\left(k_{y}, \omega\right)=\frac{1}{\Lambda} \arccos \left(\frac{A+D}{2}\right) .
$$

The modes, in which $|A+D| / 2<1$, correspond to the real $K$. If $|A+D| / 2<1$, the relation $K=m \pi / \lambda+i K_{m}$ takes place, i.e., the imaginary part in the wave vector $K$ is non-zero, and the wave is damped. Thus the so-called band-gap opens. The frequencies corresponding to the band-gap boundaries are found from the condition $|A+D| / 2=1$.

At normal incidence $\left(k_{y}=0\right)$, the dispersion dependence $\omega(K)$ has, according to (25), the following form:

$$
\cos (K \Lambda)=\cos \left(k_{1} a\right) \cos \left(k_{2} b\right)-\frac{1}{2}\left(\frac{n_{2}}{n_{1}}+\frac{n_{1}}{n_{2}}\right) \sin \left(k_{1} a\right) \sin \left(k_{2} b\right) .
$$

The quantities in Eqn. (26) have the following physical meaning: $i=1$ is the subscript related to the first medium, while $i=2$ is the subscript related to the second medium; $n_{1}=n_{1}(\omega)$ is the refractive index of the first medium, and $n_{2}=n_{2}(\omega)$ is the refractive index of the second medium; $a=(1-\eta) \Lambda, b=\eta \Lambda, \eta$ is the content of the second medium in the layered PTC; $k_{i}(\omega)=\frac{\omega \cdot n_{i}}{C_{0}}$ is the wave vector in the $i$-th medium, and $C_{0}=3 \cdot 10^{8} \mathrm{~m} / \mathrm{s}$ is the velocity of light in vacuum.

As the first approximation we can assume that the refractive index values of both layers $n_{1}$ and $n_{2}$ are the constant values and do not depend on the electromagnetic radiation frequency. At the next stage we shall take into account their dependence on the frequency (or on the wavelength of a radiation illuminating the PTC). For example, let us consider a one-dimensional PTC, one layer of which is amorphous quartz $\left(\mathrm{SiO}_{2}\right)$, and second layer is atmospheric air, for which the refractive index is $\sim 1$. Additionally, we will consider that the refractive index of $\mathrm{SiO}_{2}$ is not dependent upon the wavelength, i.e. $n_{1}=1.47$. Finally, we will use the following values of the parameters $a_{1}$ and $a_{2}$ : specifically, $a_{1}=136 \mathrm{~nm}$, and $a_{2}=48$ $\mathrm{nm}$. In this case the dispersion law for the electromagnetic waves in PTC can be obtained by using Eqn. (26). The results of numerical simulation of the dispersion curves $\omega(k)$ (these curves were taken from our study [8]) are plotted in Fig. 2 by the bold lines. As is seen from this Figure, we can discern in the spectrum three dispersion branches $\omega_{j}(k),(j=1,2,3)$, where the first one is related to the low-frequency range, including the infra-red spectral area, the second one is related to the visible spectral range, and the third one is related to the near ultra-violet spectrum. The additional branches are plotted in view of anomalous dispersion of the refractive index at approaching the electronic absorption band in $\mathrm{SiO}_{2}$. In Fig. 2 two photonic band-gaps, where the first one corresponds to the Brillouin zone boundary $\left(k=\frac{\pi}{\Lambda}\right)$, and the second one is related to the Brillouin zone center $(k=0)$, are seen. The second dispersion branch is related to the case, where the directions of the phase and group velocities are opposite with respect to one another, i.e. to the case of negative effective refractive index $(n<0)$.

As was found in study [8], the good enough description of the dispersion curves (see the gray curves (2) in Fig. 2, which were plotted on the basis of the exact theory given by Eqn. (26)), can be carried out by using the approximated formulas of the kind: 


$$
\begin{gathered}
\omega=2 \frac{C_{0}}{m_{1} \Lambda} \sin \left(\frac{k \Lambda}{2}\right),- \text { the first dirpersion branch, } \\
\omega^{2}=\omega_{2}^{2}-\left(2 \frac{C_{0}}{m_{2} \Lambda}\right)^{2} \sin ^{2}\left(\frac{k \Lambda}{2}\right) \text { - the second dirpersion branch, } \\
\omega^{2}=\omega_{3}^{2}+\left(2 \frac{C_{0}}{m_{3} \Lambda}\right)^{2} \sin ^{2}\left(\frac{k \Lambda}{2}\right) \text { - the third dirpersion branch. }
\end{gathered}
$$

Here $\omega_{1}$ and $\omega_{2}$ are the frequencies corresponding to the edges of the second order bandgap, $\mathrm{m}_{1}, \mathrm{~m}_{2}$, and $\mathrm{m}_{3}$ are the effective refractive indices for the first, the second and the third dispersion branches accordingly. The following values for the frequencies and refractive indices were taken: $\omega_{2}=3.62 \cdot 10^{15} \mathrm{radian} / \mathrm{s}, \omega_{2}=3.62 \cdot 10^{15} \mathrm{radian} / \mathrm{s}, \omega_{3}=4.14 \cdot 10^{15} \mathrm{radian} / \mathrm{s}$, and $m_{1}=0,94 ; m_{2}=0.555 ; m_{3}=0.435$. Besides, the opportunity of approximating the dispersion curves close to the center of the Brillouin zone by the so-called quasi-relativistic formulas (30) - (33) was considered:

$$
\omega=\frac{C_{0}}{m_{1}} k-\text { the first dirpersion branch, }
$$

$\omega^{2}=\omega_{2}^{2}-\left(\frac{C_{0}}{m_{2}}\right)^{2} k^{2}-$ the second dirpersion branch,

$$
\omega^{2}=\omega_{3}^{2}+\left(\frac{C_{0}}{m_{3}}\right)^{2} k^{2} \text { - the third dirpersion branch, }
$$

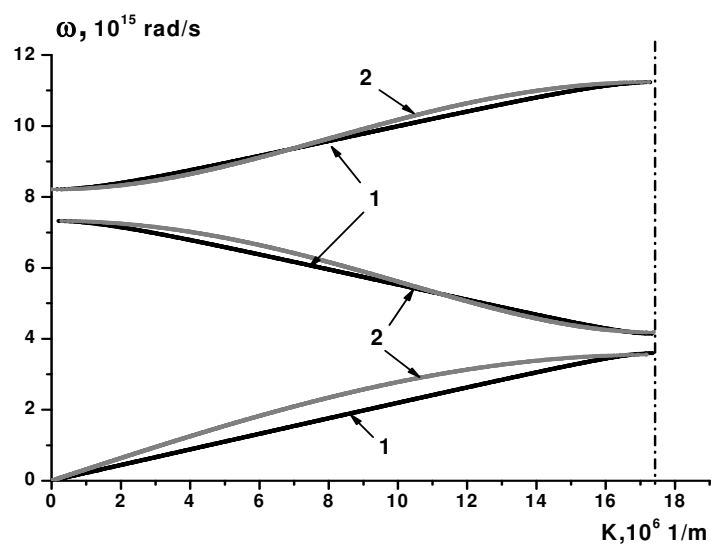

Fig. 2. The dispersion law $\omega(k)$ for a one-dimensional PTC; $(1)$ - the results of calculation of the dispersion dependence $\omega(k)$ according to (26), (2) - the results of calculation of the dispersion dependence $\omega(k)$ with the help of sinusoidal approximation. 


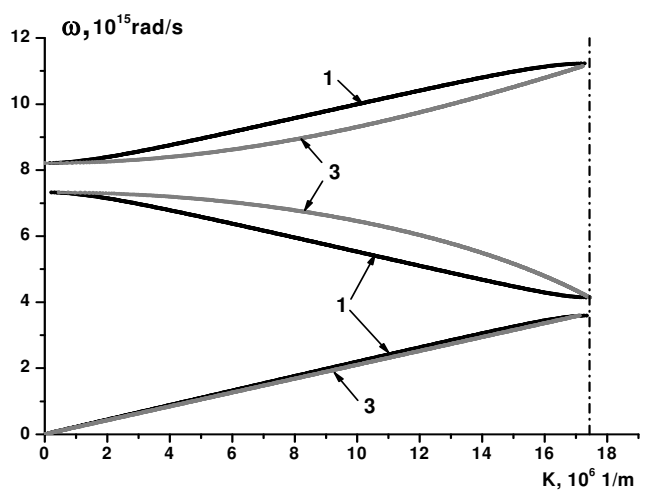

Fig. 3. The dispersion law $\omega(k)$ for a one-dimensional PTC; $(1)$ - the results of calculation of the dispersion dependence $\omega(k)$ according to (26), (3) - the results of calculation of the dispersion dependence $\omega(k)$ with the help of quasi-relativistic approximation.

As is seen from Fig. 3, the satisfactory agreement between the curves 1 and 3 takes place only at small values of a wave vector (i.e., close to the center of the Brillouin zone). Nonetheless, this approach allows us to estimate the effective photonic mass basing on the equality (here $m_{0}$ and $E_{0}$ are the effective rest mass and the rest energy of the photon.)

$$
m=\frac{1}{\frac{d^{2} E}{d p^{2}}}=\frac{\hbar}{\frac{d^{2} \omega}{d k^{2}}} ; \quad\left|m_{0}\right|=\frac{E(0)}{C^{2}}=\frac{\hbar \omega(0)}{C^{2}} .
$$

For the second dispersion branch the effective rest mass of the photon appears to be negative and equal to $m_{2}=-\frac{\hbar \omega_{1}}{\left(\frac{C_{0}^{2}}{m_{2}^{2}}\right)}=-0,13 \cdot 10^{-35} \mathrm{~kg}$. Accordingly for the third branch we obtain: $m_{3}=\frac{\hbar \omega_{2}}{\left(\frac{C_{0}^{2}}{m_{3}^{2}}\right)}=0,09 \cdot 10^{-35} \mathrm{~kg}$. Thus, the effective rest mass of a photon inside the PTC appears to be non-zero and can acquire both positive and negative magnitudes.

If one takes into account the dispersion of refractive index for the layers, forming PTC, the dispersion law $\omega(k)$ can also be received from numeric solution to Eqn. (26). For example, let us consider PTC, where the first medium is $\mathrm{SiO}_{2}$, whereas the second medium is atmospheric air or water. The refractive index of air is assumed to be equal to unity. Thus for the dependence of the $\mathrm{SiO}_{2}$ refractive index versus a wavelength we can use the formula:

$$
n_{1}^{2}-1=\frac{0,6962 \lambda^{2}}{\lambda^{2}-0,0684^{2}}+\frac{0,4079 \lambda^{2}}{\lambda^{2}-0,1162^{2}}+\frac{0,8975 \lambda^{2}}{\lambda^{2}-9,896^{2}},
$$

while the same dependence for water is given by the formula:

$$
n_{2}^{2}-1=\frac{5,667 \cdot 10^{-1} \lambda^{2}}{\lambda^{2}-5,084 \cdot 10^{-3}}+\frac{1,732 \cdot 10^{-1} \lambda^{2}}{\lambda^{2}-1,818 \cdot 10^{-2}}+\frac{2,096 \cdot 10^{-2} \lambda^{2}}{\lambda^{2}-2,625 \cdot 10^{-2}}+\frac{1,125 \cdot 10^{-1} \lambda^{2}}{\lambda^{2}-1,074 \cdot 10^{1}}
$$




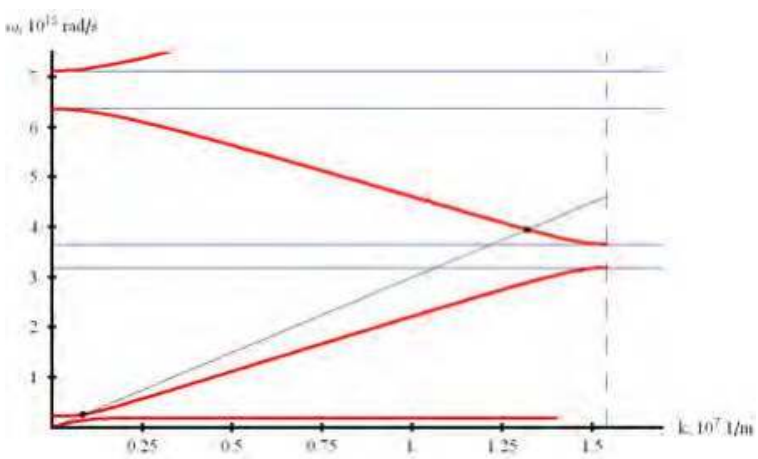

(a)

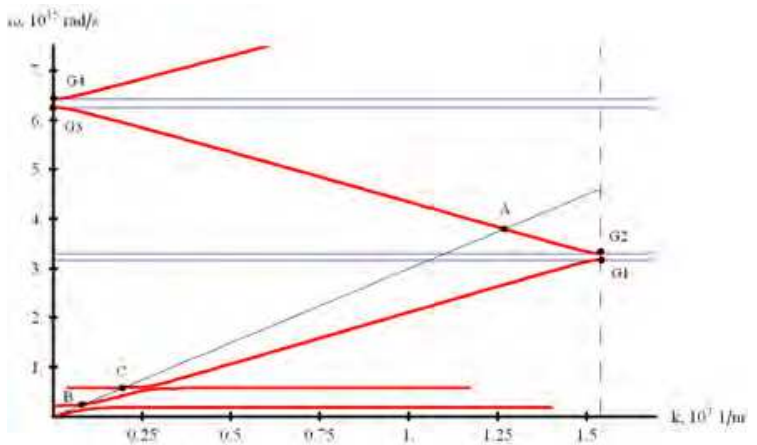

(b)

Fig. 4. The calculated dispersion curves; (a) - the initial PTC; (b) - the PTC, filled with water. The band-gap boundaries (thin solid lines), the edge of the first Brillouin zone together with the straight line indicating the dispersion law for a light wave in vacuum $\left(\omega=C_{0} k\right)$ are indicated.

The calculated dispersion curves for these cases are shown in Fig. 4 (a) and (b). As is seen in this Figure, implantation of water instead of air in PTC results in decreasing the optical contrast and, accordingly, in reducing the band-gap width for the visible and ultraviolet spectral ranges. Accounting for the dispersion of the refractive index for $\mathrm{SiO}_{2}$ results in occurrence of the additional dispersion branch in the infrared spectral range; this branch is related to the polariton curve, stimulated by the polar vibrations, e.g., the vibrations along the bond $\mathrm{Si}-\mathrm{O}$ in the microstructure of quartz. In Fig. 4 the points of intersection of the straight line, corresponding to the light wave (this line is set by the formula $\omega=C_{0} k$, for which the effective refractive index is equal to unity) are marked. Thus according to the known Fresnel formulas the reflectance of a light wave from the PTC interface approaches zero, and the material should become absolutely transparent (provided that the absorption is absent).

\subsection{Calculation of the dispersion characteristics for the one-dimensional PNC}

As was already noted, PNC can either be considered as PTC with making allowance for the fact that the sonic wave velocities depend upon the type of material of a layer. Using the 
optical-acoustic analogy for describing the dispersion of acoustic waves in PNC and basing upon Eqn. (26), we obtain the following dispersion equation for the acoustic wave propagating in PNC along the crystallographic direction (111):

$$
\cos (k a)=\cos \left(k_{1} a_{1}\right) \cdot \cos \left(k_{2} a_{2}\right)-\frac{1}{2} \frac{V_{1}^{2}+V_{2}^{2}}{V_{1} \cdot V_{2}} \sin \left(k_{1} a_{1}\right) \cdot \sin \left(k_{2} a_{2}\right) .
$$

The quantities entering into (34) have the following physical meaning: $i=1$ is the subscript for $\mathrm{SiO}_{2}$ (opal matrix); $i=2$ is the subscript for the layer, filled with a metal or liquid; $V_{1}$ is the velocity of acoustic waves in opal; $V_{2}$ is the velocity of sound in the medium that fills the pores in the opal (see table 1 ); $\eta=0,26$ is the effective sample porosity coefficient, $D=220$ $\mathrm{nm}$ is the diameter of the quartz globules; $a=D \sqrt{2 / 3}$ is the period of the structure of the opal samples under investigation; $a_{1}=(1-\eta) a, a_{2}=\eta a ; \omega_{i}$ is the cyclic frequency of the acoustic wave; $k_{i}(\omega)=\omega / v_{i}$ is the wave vector in the $i$-th medium.

\begin{tabular}{|c|c|c|}
\hline Material & Transverse wave velocity, $\mathrm{km} \cdot \mathrm{s}^{-1}$ & Longitudinal wave velocity, $\mathrm{km} \cdot \mathrm{s}^{-1}$ \\
\hline Opal & 3.3 & 5.3 \\
\hline Air & 0.1 & 0.3 \\
\hline Water & 0.6 & 1.5 \\
\hline Gold & 1.2 & 3.2 \\
\hline
\end{tabular}

Table 1. Velocities of longitudinal and transverse acoustic waves

Based on the numerical analysis of (34), we constructed the dispersion dependences $\omega(k)$ for different branches in the acoustic region of the spectrum. The numerically simulated acoustic properties of various PNCs are shown in Fig. 5.

The abscissas are the wave vector values, scaled in $\mathrm{m}^{-1}$, and the ordinates are the cyclic frequencies $\left(\mathrm{rad} \cdot \mathrm{s}^{-1}\right)$; the solid lines indicate longitudinal waves and the dashed lines indicate transverse waves. Fig. 5 (a) corresponds to the initial (unfilled) opal containing air in its pores, whereas Fig. 5 (b) shows the dispersion dependence $\omega(k)$ for the sample with water in its pores, and Fig. 5 (c) displays acoustic branches of PTC with nanoparticles of gold. As is seen from these graphs, both in PTC and PNC the band-gaps are being formed, whose location and bandwidth depend on the spatial period of the superstructure and the type of material. The dispersion dependence of quasi-particles traveling along the crystalline lattice can be found from the known relation $[6,7]$ :

$$
V_{g r}(\omega)=\frac{d \omega(k)}{d k}=\frac{1}{\frac{d k(\omega)}{d \omega}}
$$

The corresponding dependences of group velocities are shown in Fig. 6. Figures 6 (a) - (c) correspond to the initial PNC, to the crystal filled with water, and to the opal filled with gold, respectively. Note that the group velocity of the acoustic waves becomes zero at the boundaries of the band-gaps. Besides, at the band-gap boundaries the group velocity of the acoustic phonons approaches zero, which corresponds to "stopping" of phonons at the corresponding frequencies. Such phenomenon is quite similar to "stopping" of photons, related to the band-gap boundaries of PNC. 


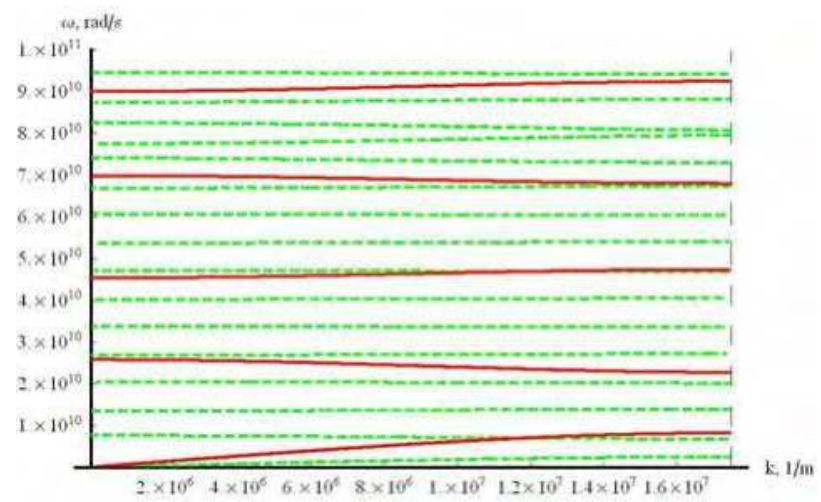

(a)

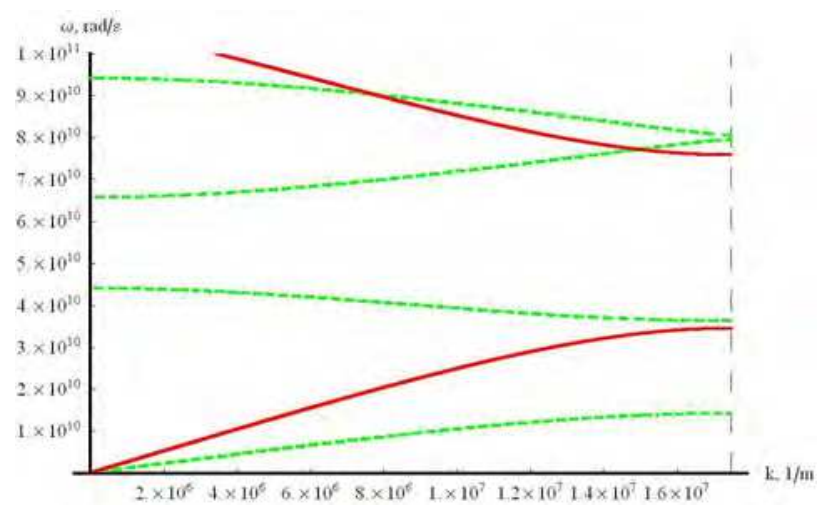

(b)

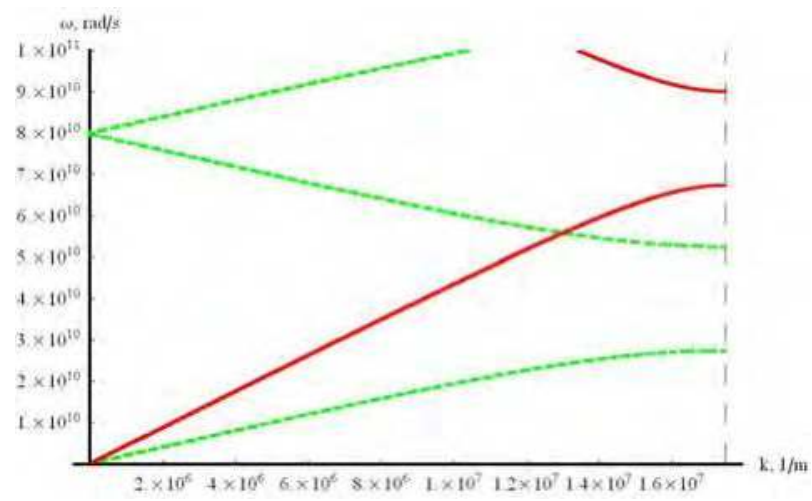

(c)

Fig. 5. Dispersion dependence $\omega(k)$ for three types of PNCs: (a) the initial PNC, (b) the PNC, filled with $\mathrm{H}_{2} \mathrm{O}$, (c) the PNC with Au nanoparticles. Solid and dashed curves correspond to longitudinal and transverse waves, respectively. 


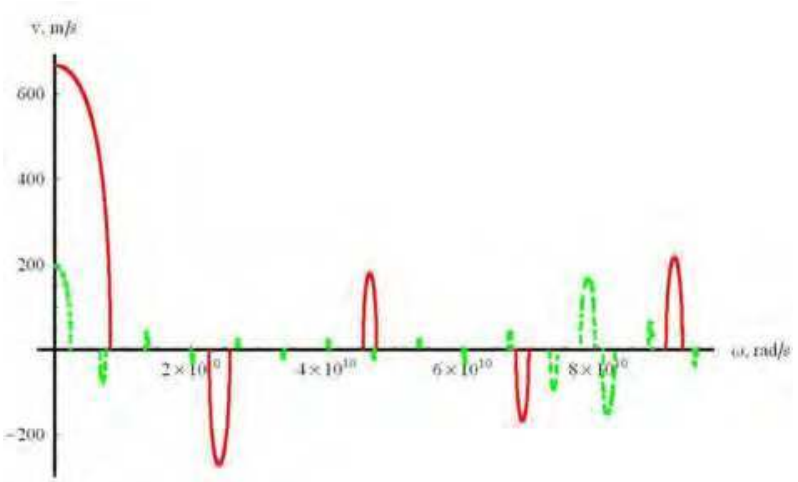

(a)

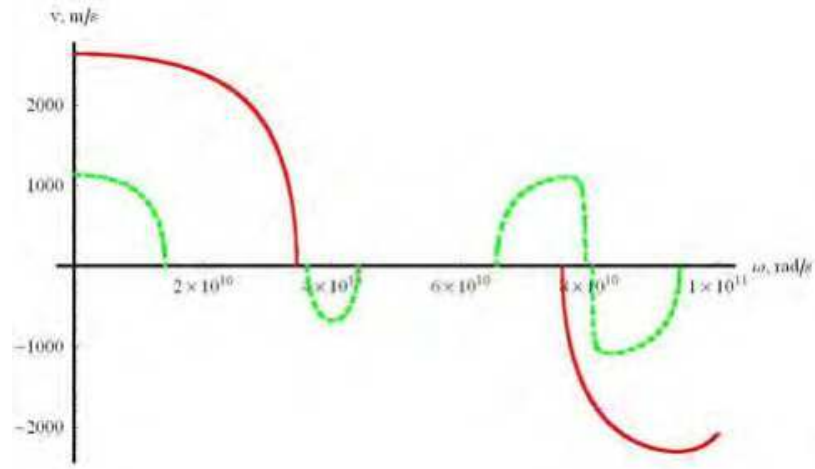

(b)

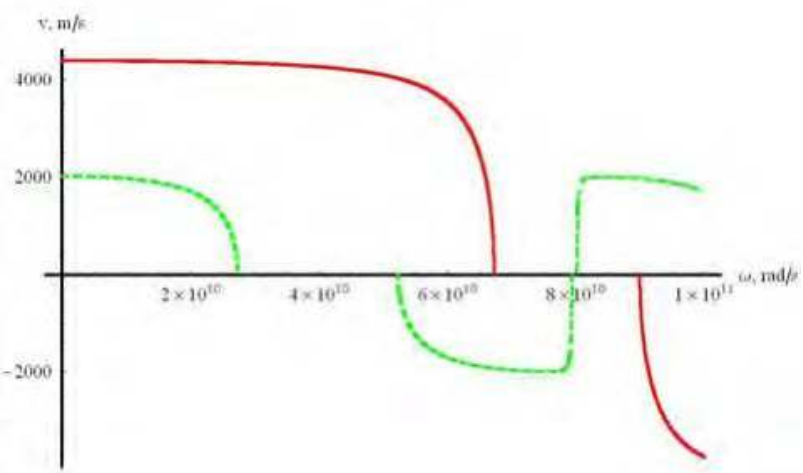

(c)

Fig. 6. Group velocity of phonons in the investigated samples: (a) the initial PNC, (b) the PNC filled with water, (c) the PNC filled with gold. Solid and dashed curves correspond to longitudinal and transverse waves, respectively. 


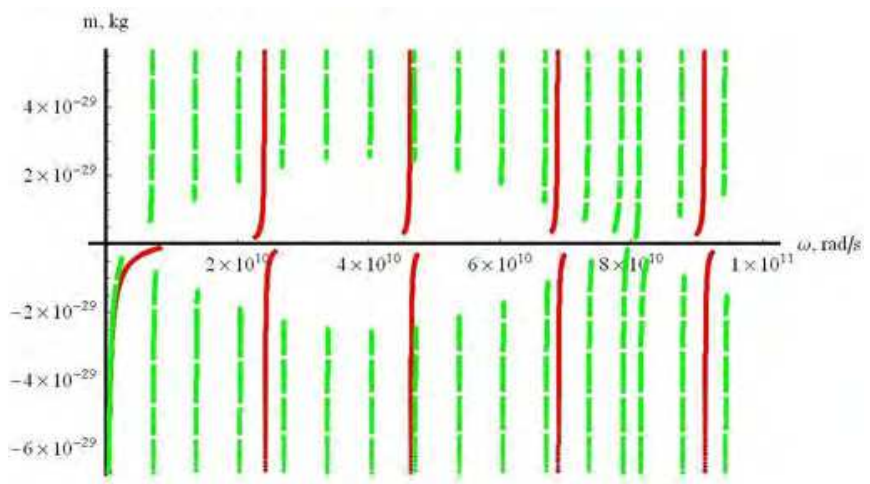

(a)

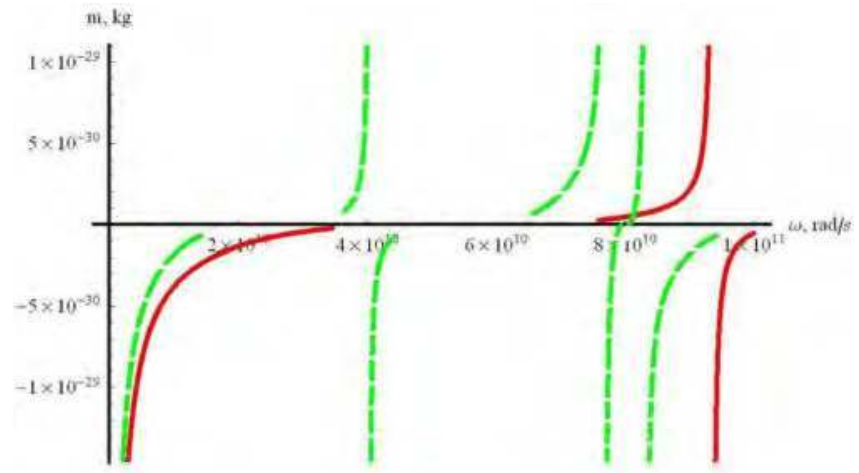

(b)

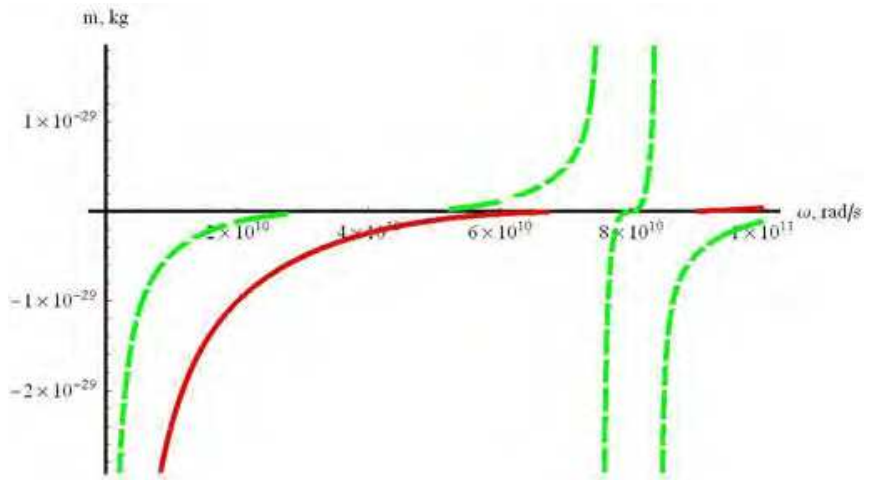

(c)

Fig. 7. Mass of acoustic quasi-particles for different types of opal: (a) initial opal, (b) opal with water, (c) opal with gold. Solid and dashed curves correspond to longitudinal and transverse waves, respectively. 
According to the general definition of the effective mass of a quasi-particle [6, 7], the effective mass of acoustic phonons can be calculated by the formula

$$
m(\omega)=\hbar\left[\frac{d^{2} \omega(k)}{d k^{2}}\right]^{-1}=\frac{\hbar}{V_{g^{r}}(\omega)\left[d V_{g^{r}}(\omega) / d \omega\right]} .
$$

This effective mass is related to slow acoustic waves and is many orders of magnitude smaller than the mass of photons in PNC, and can be estimated from the relation $\left|m_{0}\right|=\frac{\hbar \omega(0)}{S^{2}}$, where $S$ is corresponding sonic velocity. In particular, the effective mass of the transverse acoustic phonons related to the second dispersion branch of PNC containing the atmospheric air (see Fig. 6) is equal to $m_{0}=-24 \cdot 10^{-30} \mathrm{~kg}$; for PNC containing water we have $m_{0}=-3,64 \cdot 10^{-30} \mathrm{~kg}$; and for PNC containing gold we obtain $m_{0}=-6,94 \cdot 10^{-30} \mathrm{~kg}$. Accordingly for the third dispersion branch the effective mass appears to be positive and slightly exceeds (by the absolute value) the indicated above values of the effective rest mass of phonons.

Summarizing, in PNC the acoustic phonons possess by the rest mass; the phonon rest mass by its absolute value is $5-6$ orders of magnitude less than the effective rest mass of photons in PTC, and can be both positive and negative.

\subsection{Structure and the techniques of preparation of the globular photonic crystals}

The important example of the three-dimensional PTC (PNC) is the so-called globular photonic crystal composed of densely packed balls (globules) as the face-centered cubic crystal lattice. The diameter of the globules is slightly changed within the whole structure of a crystal. Depending on the technological process this diameter can vary within the range of $200-1000 \mathrm{~nm}$. To the present time the globular photonic crystals composed of the balls of synthetic opal $\left(\mathrm{SiO}_{2}\right)$, titanium oxide $\left(\mathrm{TiO}_{2}\right)$, and Polystyrene are known. There exist the voids (pores) between the globules of a photonic crystal, which can be filled with some foreign additives. For example, it is possible to implant into the pores of a globular phoptonic crystal some liquids, which moisturize the globule interface, and solid dielectrics, including piezoelectrics and ferroelectrics. Besides, it is possible to implant magnetic

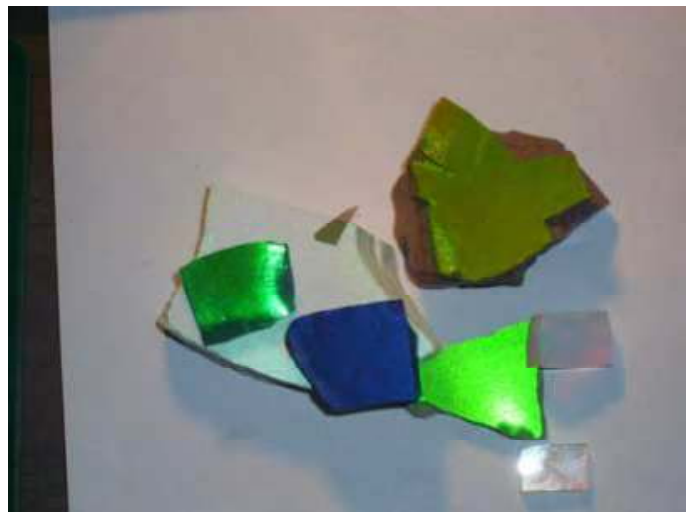

Fig. 8. Samples of 3D-PTC, obtained from the synthetic opals under different technological conditions. 
materials, semiconductors, metals and superconductors. Thus, we have a wide opportunity to create new materials of a hybrid-like type: dielectric-ferroelectric, dielectric-magnetic, dielectric-metal etc. We also can control the dielectric, acoustic and galvanic properties of such hybrid materials by changing the diameter of globules.

Some samples of three-dimensional PTCs under study are illustrated in the photo, see Fig. 8 . The white large sample (at the foot of the photo) was annealed in the atmospheric air at the temperature of $600 \mathrm{C}$. Color (green and blue) samples were annealed in the atmosphere of argon. During the process of growth and annealing these samples were saturated by carbon as the result of destruction of organic molecules, which were initially (in the trace amounts) located in the samples.

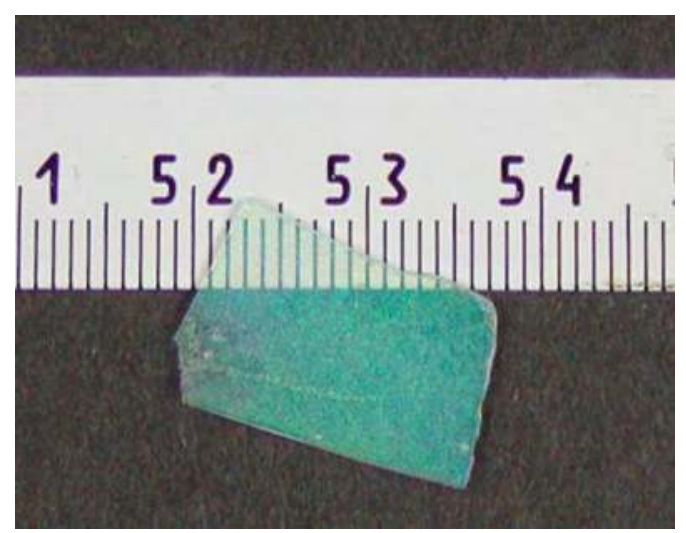

(a)

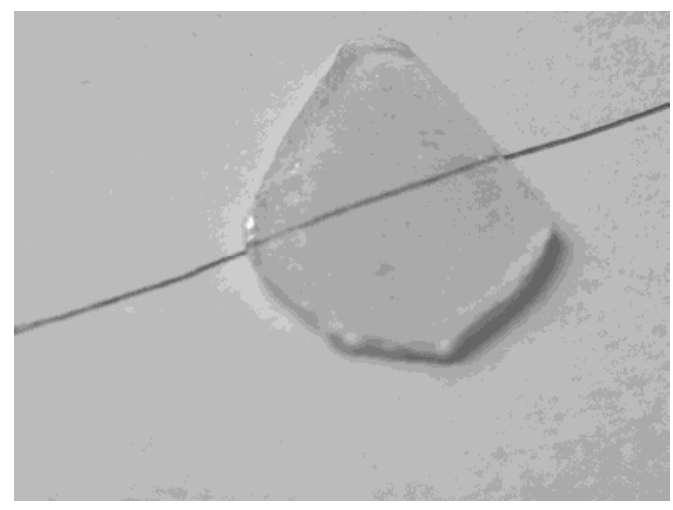

(b)

Fig. 9. PTC, transparent in the visible spectral range; (a) - PTC, containing the quantum dots. This sample was filled by $\mathrm{ZrO}_{2}$ nanoparticles and then was subjected to annealing at high temperature (up to $1200 \mathrm{C}$ ); as the result, the sample became transparent as the size of implanted inclusions of $\mathrm{ZrO}_{2}$ nanoparticles was essentially less than the photonic crystal lattice constant and a wavelength in the visible range. (b) - PTC, filled with glycerol-water mixture. 


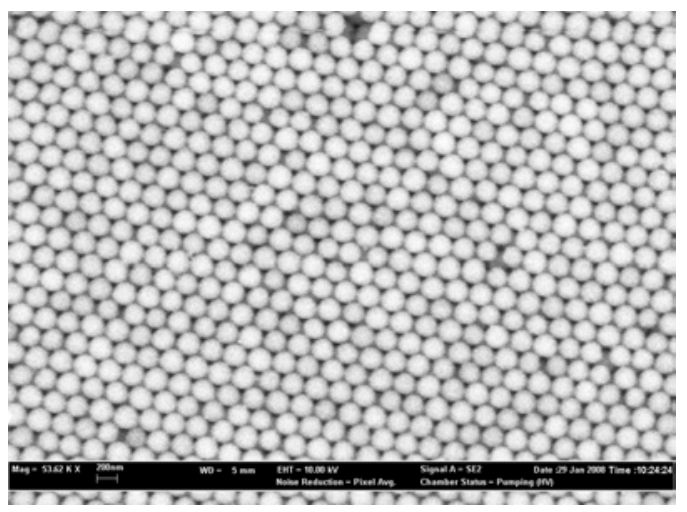

(a)

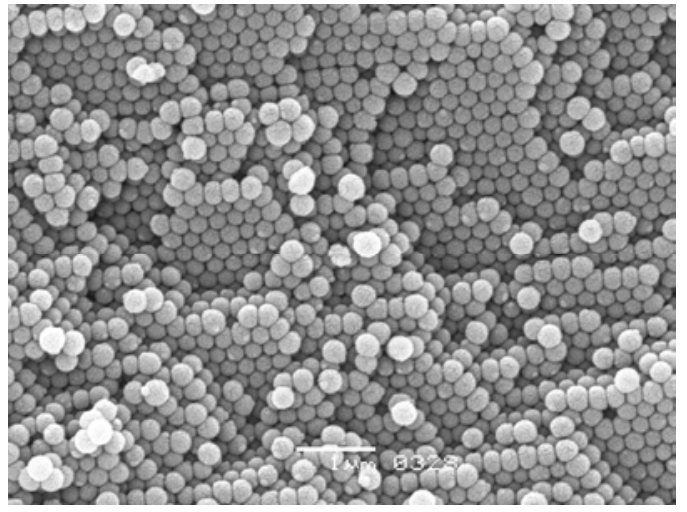

(b)

Fig. 10. The images of (111) surfaces for two ((a) and (b)) investigated synthetic opals, obtained with the help of electronic microscope.

Electronic images of the globular PTC surface (111) for two investigated samples are shown in Fig. 10 (a) and (b). We can see that the nanostructure of sample in Fig.10 (a) is close to the ideal one. In the case of the second sample (Fig. 10 (b)) there exist numerous defects arisen due to certain disordering processes. Initial synthetic opals have been filled with some organic (Stilbene, glycerol, acetone, nitrobenzene) or inorganic (sodium nitrite, sulfur, $\mathrm{ZrO}_{2}$ ) chemicals. At the certain concentration of glycerol-water mixture its refractive index appeared to be very close to that for a quarts globule. In this way almost transparent 3DPTC have been obtained (see Fig. 9 (b)).

The processes of the opal sample processing are shown in Fig. $11(\mathrm{a}, \mathrm{b})$. We have implanted nanoparticles of some metals ( $\mathrm{Au}, \mathrm{Ag}, \mathrm{Ga}$ ) into the photonic crystal pores localized between the globules. The sample was filled with $\mathrm{ZrO}_{2}$ nanoparticles and then was subjected to annealing at high temperature (up to $1200 \mathrm{C}$ ); as the result, the sample became transparent as the size of implanted inclusions of $\mathrm{ZrO}_{2}$ nanoparticles was essentially less than the photonic crystal lattice constant and the visible range wavelength. Accordingly, such spatial arrangement of inclusions can be described as the array of spatially ordered quantum dots 
in the transparent crystal of quartz. The schematic nanostructure of such quantum dots in PTC is illustrated in Fig. $11 \mathrm{c}$.
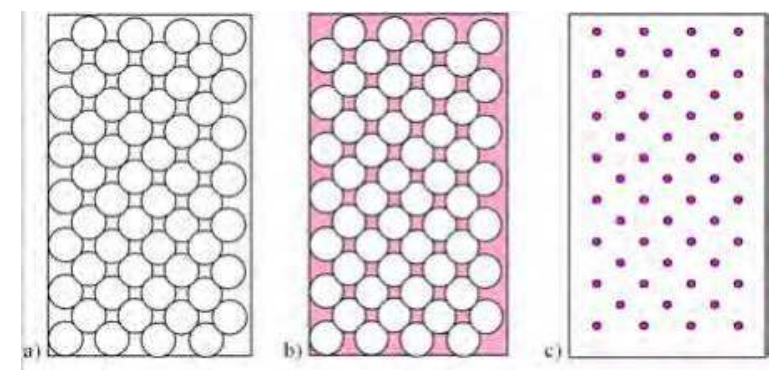

Fig. 11. Structures of 3D-PTC filled by dielectrics or metals; (a) - initial synthetic opal, (b) - opal, filled with some substance, (c) - result of the high temperature annealing of the sample, containing the particles of $\mathrm{ZrO}_{2}$, whose melting temperature is higher than that for quartz.

\subsection{Optical properties of the globular PTC}

In what follows we will analyze the optical and acoustic properties of globular PTC; it is clear that we can describe the both properties in the framework of the same approach. This is why the following considerations basically repeat the models applied above, but now we should bear in mind that we deal with the three-dimensional periodic medium. Assuming that the light wave is directed along the (111) vector in a crystal, it is still possible to use the approximation of effective one-dimensional model of the layered PTC [6, 7]. In this case the dispersion law of the globular PTC on the basis of the synthetic opal, whose pores are filled with atmospheric air, is given by the following formula, which is quite similar to Eqn. (34) for the dispersion law of acoustic waves in the layered PTC:

$$
\cos k_{1} a_{1} \cdot \cos k_{2} a_{2}-\frac{1}{2} \frac{\varepsilon_{1}+\varepsilon_{2}}{\sqrt{\varepsilon_{1} \cdot \varepsilon_{2}}} \sin k_{1} a_{1} \cdot \sin k_{2} a_{2}=\cos k a .
$$

The parameters here are the following: $\varepsilon_{1}$ is the dielectric permittivity of quartz (naturally for the oprtical range of frequencies); $\varepsilon_{2}$ is the dielectric permittivity of air, $a_{1}=(1-\eta) a$, $a_{2}=\eta a$, where $\eta$ is the effective sample porosity, $a=D \sqrt{2 / 3}$ is the period of the structure of the sample, $D$ is the effective diameter of quartz globule, $\omega_{i}$ is the cyclic frequency of the electromagnetic wave, $k_{i}(\omega)=\frac{\omega}{c_{0}} \sqrt{\varepsilon_{i}}$ is the wave vector in $\mathrm{SiO}_{2}(i=1)$ and in the air $(i=2)$.

In Fig. 12 the dispersion dependence $\omega(k)$ for the incident (along the direction (111)) electromagnetic wave in the globular PTC, whose pores filled with atmospheric air, and the effective globule diameter is $D=225 \mathrm{~nm}$. The Fig. 13 illustrates the two-branch dependence $\omega(k)$ for the globular PTC filled with the liquid having the refractive index close to that for $\mathrm{SiO}_{2}$. As is seen from the graphs, in that case the band-gap width approaches zero.

Figs. 14 and 15 illustrate the dispersion law $\omega(k)$ of electromagnetic waves for the globular PTC, filled with the dielectric or metal accordingly. Figs. 16 and 17 show the character of changing the dispersion law owing to the occurrence of the low and high frequency 


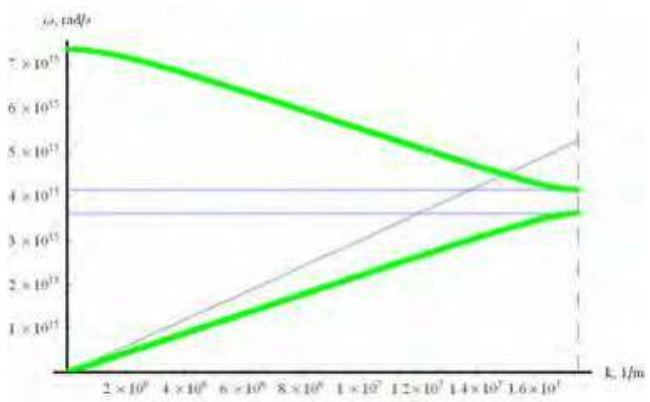

Fig. 12. The dispersion curves $\omega(k)$ for the first two branches of the globular PTC filled with air. The straight line obeys the dispersion law in vacuum.

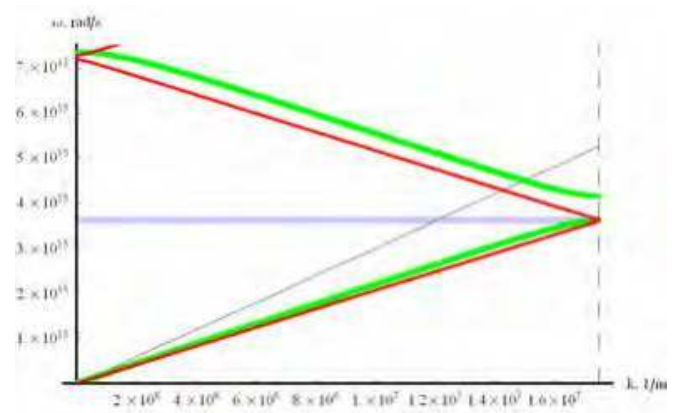

Fig. 13. The dispersion curves $\omega(k)$ for the first two branches of the globular PTC, filled with water. The upper curve corresponds to the initial (free of water) crystal, the lower curve corresponds to the crystal, whose pores contain a liquid with the refractive index close to that for quartz.

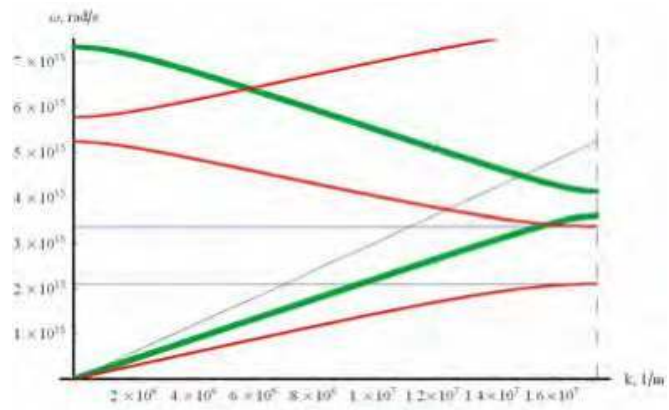

Fig. 14. The dispersion curves $\omega(k)$ for the first two branches of the globular PTC, filled with the dielectric. 


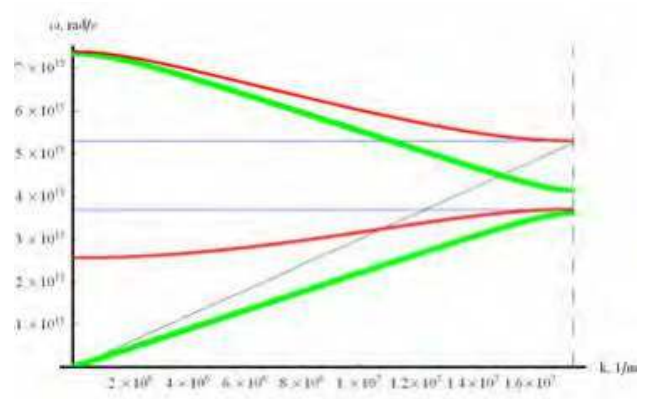

Fig. 15. The dispersion curves $\omega(k)$ for the first two branches of the globular PTC, filled with the metal.

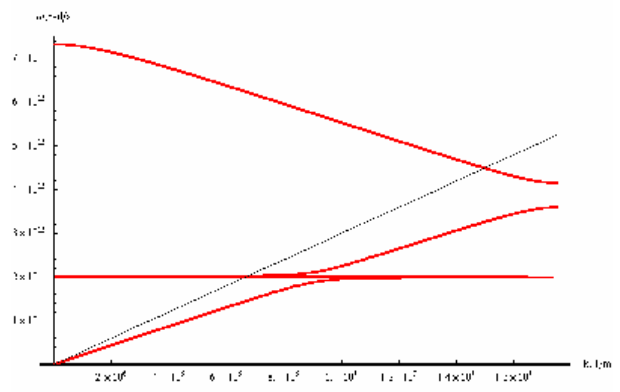

Fig. 16. The dispersion curves $\omega(k)$ for the first two branches of the globular PTC for the case, where the low-frequency resonance exists.

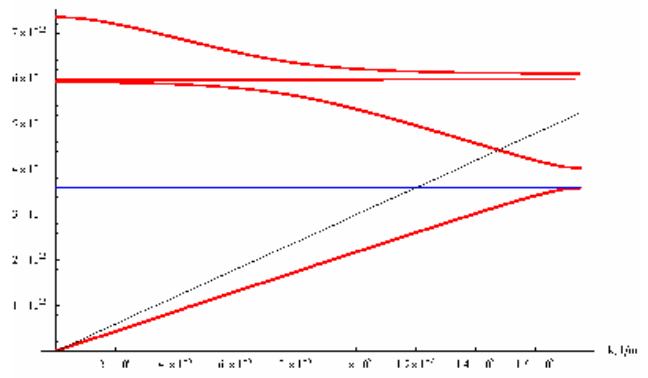

Fig. 17. The dispersion curves $\omega(k)$ for the first two branches of the globular PTC for the case, where the high-frequency resonance exists.

resonances accordingly; the resonances arise due to adding the certain substance in the pores. As is seen from Figs. 13 - 15, the implantation of dielectrics, whose refractive index exceeds that of quartz, into the pores of the globular PTC results in changing the width of the band-gap and its shifting to lower frequencies. At the same time, at implanting metal into these pores the band-gap shifts to higher frequencies, see Fig. 15. If one implants the 
substance, characterizing by the presence of resonances close to the band-gap spectrum, the dispersion curves $\omega(k)$ drastically change; it becomes possible that new band-gaps are being formed, and this process is essentially dependent on the resonant frequencies of the implanted substance, see Figs. 16, 17.

The implantation of various chemicals into the globular PTC was carried out by various techniques: among these was impregnation by a liquid wetting quartz, saturation of the crystal matrix by solutions of various salts with subsequent annealing, and also some laser methods including ablation. To analyze the spectra of reflectance of incident broadband electromagnetic radiation from the globular PTC interface, whose pores contain various substances, the experimental setup (see Fig. 18) was designed; its characteristics are described in Ref. [9]. In this setup the radiation of halogen or deuterium lamp (14) was directed with the help of an optical fiber probe perpendicular to the crystal interface (3). The optical fiber diameter was $100 \mu \mathrm{m}$, and the spatial resolution of the setup was on the level of $0.2 \mathrm{~mm}$. With the help of another optical wave-guide the oppositely reflected radiation was

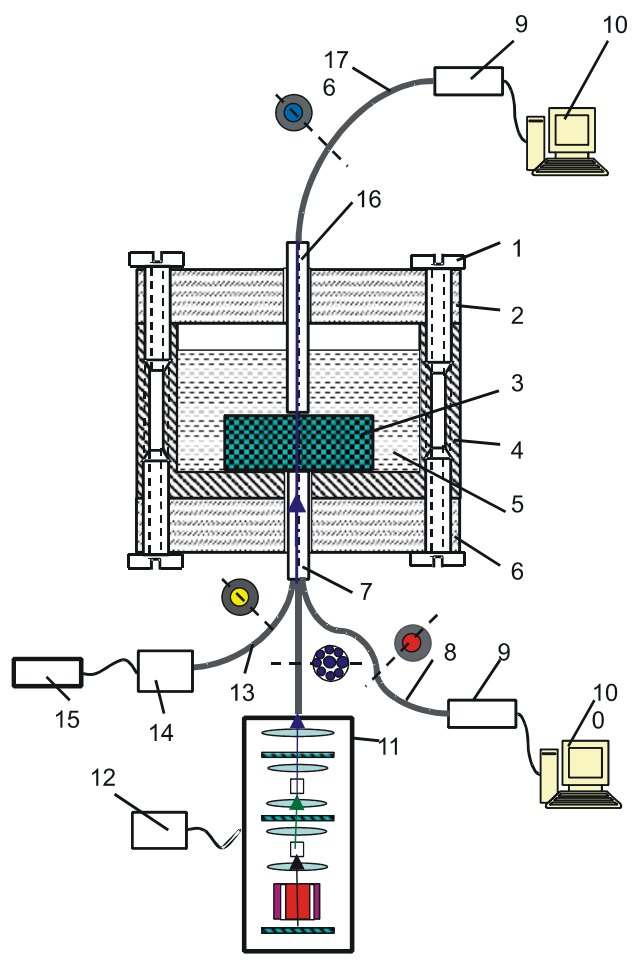

Fig. 18. The schematic of the experimental setup for analyzing the spectra of radiation reflected from the PTC interface; (1) - screws; (2) - the top Teflon cover-sheet; (3) - the PTC; (4) - the cell; (5) - the liquid sample; (6) - the bottom Teflon cover-sheet; (7) - the optical fiber probe; (8) - the wave-guide; (9) - the mini-spectromemer; (10) - the computer; (11) the YAG:Nd ${ }^{3+}$ - laser; (12) - the power supply unit for the wave-guides; (13) - the waveguide; (14) - the halogen lamp; (15) - the power supply unit for the lamp; (16) - the optical fiber probe for investigating the transmission spectra; (17) - the wave-guide. 
input to a mini-spectrometer FSD-8, where the reflectance spectra in the range of $200-1000$ $\mathrm{nm}$ were processed in the real time. The spectral resolution of the reflectance spectra was $\leq 1$ $\mathrm{nm}$. Using the laser radiation (pulse repeating YAG: $\mathrm{Nd}^{3+}$ laser with the possibility of doubling or quadrupling the frequency of the radiation) allowed us to carry out additional implantation of dielectrics or metals into the pores of the crystal with the simultaneous controlling the spectrum of the band-gap (this spectrum depends on the type and amount of the implanted substance). Using the additional optical fiber probe (16) allowed us to analyze the transmission spectrum with the help of second mini-spectrometer (9). The experimental data were input to the analog-to-digital converter of the computer (10) for the final processing.

In Fig. 19 the reflectance spectra of the globular PTC with various globule diameter and containing the atmospheric air (curve 1 in Fig. 19 (a) - (c)), and water (curve 2 in Fig. 8 (a) (c)) are given. It is seen that at increase of the globule diameter, and at implantation of water into the pores the reflectance peak corresponding to the band-gap is shifted to higher frequencies. This experimental result is in agreement with formulas (38) and (39), which are relevant for the PTC model in question:

$$
\begin{gathered}
\lambda_{\text {max }}=2 \sqrt{\frac{2}{3}} D \sqrt{n_{e f f}^{2}-\sin ^{2} \theta}, \\
n_{\text {eff }}=\sqrt{n_{1}^{2} \beta+n_{2}^{2}(1-\beta)} .
\end{gathered}
$$
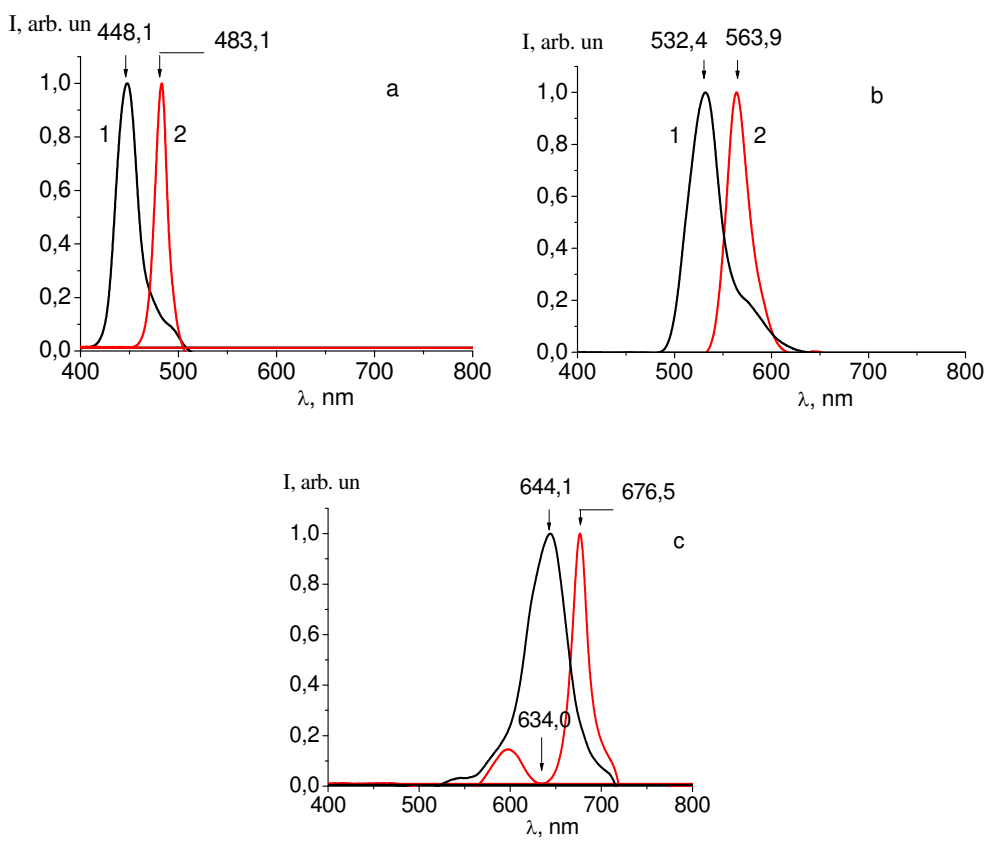

Fig. 19. The spectra of radiation reflected from (111) interface of the globular PTC with various globule diameters: $D=200(\mathrm{a}), 240$ (b) and $290 \mathrm{~nm}$ (c). 
Here $\theta$ is the angle of the radiation incidence onto the interface (111) of the PTC, $D$ is the globule diameter, and $n_{1}, n_{2}$ are the refractive indices of $\mathrm{SiO}_{2}$ and an implanted substance respectively.

As is seen in Fig. 19, the impregnation of the crystal matrix by water results in narrowing the band-gap. This is in conformity with the optical contrast decrease at approaching the refractive indices $n_{2}$ and $n_{1}$ to one another, see Eqn. (40) for the band-gap width.

$$
\Delta \lambda=\lambda_{\max } \frac{4}{\pi} \frac{\left|n_{2}-n_{1}\right|}{\left(n_{2}+n_{1}\right)} .
$$

Fig. 20 illustrates the reflectance spectrum for the first and the second band-gap. According to Eqn. (38) the frequency of the reflectance spectral maximum should belong to the visible range, and for the second band-gap that frequency should be duplicated. As is seen in this Figure, the additional reflectance peak is indeed observed in the near ultra-violet range. The curve (1) in this Figure characterizes the parameters of the second band-gap. It is noteworthy that spectral boundaries of this band-gap are shifted towards larger wavelengths. This result is due to the growth of refractive index of $\mathrm{SiO}_{2}$ in the ultra-violet spectral range.

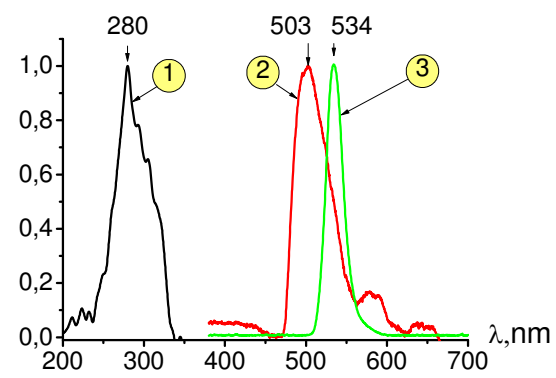

Fig. 20. The reflectance spectra of the globular PTC, filled with air (curve (2)) and water (curves (1) and (3)). The curves (2) and (3) are related to using the halogen lamp with a broad bandwidth in the visible range. The curve (1) is related to using the deuterium lamp with a broad bandwidth in the ultra-violet range.

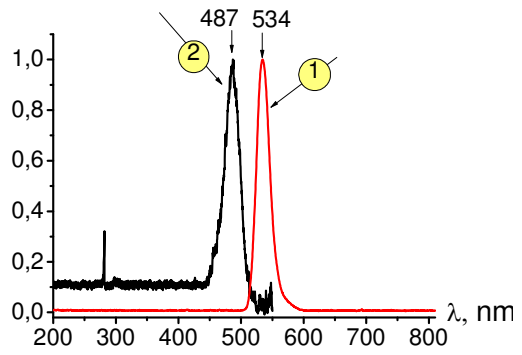

Fig. 21. The reflectance spectrum for the initial PTC (the curve 2), and the PTC doped with the nanoparticles of gold (the curve 1). 
Fig. 21 shows the reflectance spectra for the same geometry of an incident light (the radiation is reflected from (111) surface of the crystal), but now the golden particles are implanted into the crystal pores by the technique of laser ablation. As is seen in this Figure, the implantation of metal into the pores results in shifting the band-gap to higher frequencies, which is due to the fact that the real part of the metal dielectric permittivity in the range of optical frequencies is negative.

\subsection{Acoustic properties of globular PNC}

Basing on the classical Lamb model, in Refs. [6, 7] the theory of natural oscillations (modes) of isolated isotropic spherical globules was developed. In this theory the existence of two kinds of globular oscillations (modes), characterized by the subscripts $l$ and $n$, was predicted. For describing these modes the following dimensionless values were introduced:

$$
\xi_{n l}=\frac{\pi v_{n l} D}{V_{L}}, \quad \eta_{n l}=\frac{\pi v_{n l} D}{V_{T}}
$$

Here $V_{L}$ and $V_{T}$ are the velocities of longitudinal and transverse acoustic waves accordingly, $D$ is the diameter of globules, $v_{n l}$ are the corresponding frequencies in Hz. The equation for the eigenvalues $\xi_{n l}$ and $\eta_{n l}$ related to the oscillating modes, which are induced in a sphere, has the form:

$$
\begin{aligned}
& 2\left[\eta^{2}+(l-1)(l+2)\left(\frac{\eta j_{l+1}(\eta)}{j_{l}(\eta)}-(l+1)\right)\right] \frac{\xi j_{l+1}(\xi)}{j_{l}(\xi)}-\frac{1}{2} \eta^{4}+ \\
& +(l-1)(2 l+1) \eta^{2}+\left[\eta^{2}-2 l(l-1)(l+2)\right] \frac{\eta j_{l+1}(\eta)}{j_{l}(\eta)}=0
\end{aligned}
$$

where $\eta$ and $\xi$ are the corresponding eigenvalues, and $j_{l}(\eta)$ is spherical first order Bessel function. The solution to this equation gives the following relationship between the frequencies:

$$
v_{n l}=\frac{v_{0}(n, l)}{D},
$$

where $v_{0}(n, l)$ is some function, dependent upon the numbers $n$ and $l$.

The modes characterized by even numbers $n$ and $l$ are the Raman-active ones, and thus can contribute to the spectra of two-photon light scattering (by contrast to the libration modes, which cannot be displayed in the two-photon processes due the rules of selection). The equation (43) was analyzed in Refs. [6,7] for the spherical globules made of quartz; the velocities $V_{L}=5279 \mathrm{~m} / \mathrm{s}$ and $V_{T}=3344 \mathrm{~m} / \mathrm{s}$ for the longitudinal and transverse sound velocities in the amorphous quartz were substituted in the corresponding equations. The calculated values of the frequencies in the $\mathrm{GHz}$ frequency range for some globular modes are the following:

$$
v_{10}=2.617 / D=0.44 \mathrm{~cm}^{-1}, v_{20}=4.017 / D=0.68 \mathrm{~cm}^{-1},
$$

where $D=200 \mathrm{~nm}$, which is in a good conformity with the experimental data, see below. Thus, in the case of the opal matrixes the nano-sized spherical globules play a role of 
vibrating molecules. The standing waves are induced in each globule of the crystal. The pulsating modes arising in the PNC globules are related to the movements, resulting in the change of the globule material density. This is why the vibrating excitation of one particular globule can transfer to another globule; accordingly the excitation wave of the globules can travel along the crystal. As is known, it is possible to observe various kinds of non-elastic scattering in medium, e.g., the Raman scattering, the Brillouin scattering, the Bragg scattering etc. In the case of Raman scattering the oscillatory quanta corresponding to the molecular vibrations are excited (or damped). Thus if we deal with PNC, the globules with the size of several hundred of nanometers play a role of vibrating molecules. Accordingly, the non-elastic scattering of light caused by the excitations of radial vibrations of the globules was termed as Globular Scattering (GS) of light. At low intensities of incident radiation this scattering is of spontaneous character. In Ref. [10] the spectra of spontaneous GS in the synthetic opals were for the first time observed at irradiation of a CW Ar $\mathrm{Ar}^{++}$- laser with the wavelength of $514.5 \mathrm{~nm}$ in the back-scattering geometry. For such measurements the synthetic opals having the effective sphere diameter $D=204,237,284$ and $340 \mathrm{~nm}$ were used.

The GS spectrum investigated in this work consisted of six well-pronounced Stokes and antiStokes spectral peaks, whose frequencies could be associated with the resonant globular modes belonging to the range of $7-27 \mathrm{GHz}$. The presence of the anti-Stokes satellites is explained by a high "population density" of low vibration states at room temperature. As was found out, the frequencies and relative intensities of the satellites do not depend on the polarization and the angle of incidence of the radiation. Besides, these parameters did not change at rotating the sample around the normal axis in the point of incidence of laser radiation. In Ref. [10] the dependence of frequency of various acoustic modes upon the sphere diameter was studied. As against to the spontaneous Brillouin scattering, GS can be observed both in the "forward" and "backward" geometry. The frequency shift for GS appears to be essentially smaller than that for Raman scattering caused by the molecular vibrations.

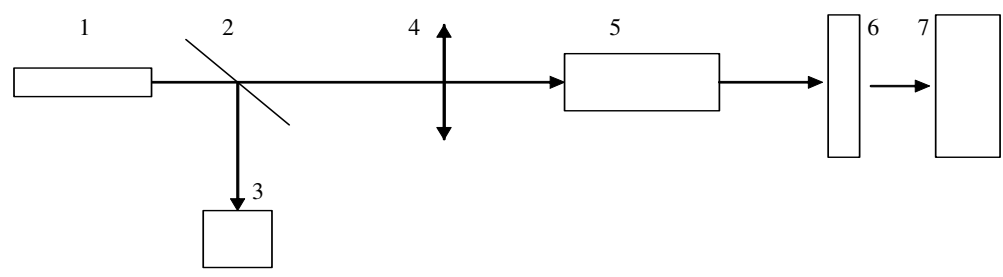

Fig. 22. The schematic of experimental setup for observing the Stimulated Globular Scattering (SGS) in the "forward" geometry; 1 - Ruby laser, 2 - half-transparent mirror, 3 power meter, 4 - focusing system, 5 - the sample under study, 6 - the Fabri-Perot interferometer, 7 - mini-spectrometer

The experiments to observe the Stimulated Globular Scattering (SGS) in the PTC were first described in [6]. The schematic of experimental setup for observing this scattering in the "forward" and "backward" geometry is illustrated in Figs. 22 and 23 accordingly. Here the pulsed Ruby laser with the wavelength of $694.3 \mathrm{~nm}$, the bandwidth $0.015 \mathrm{~cm}^{-1}$, the pulsewidth of $20 \mathrm{~ns}$, and the pulse energy of $0.4 \mathrm{~J}$ was used. The laser radiation was directed with the help of focusing lens system 4 (Fig. 22) or 6 (Fig. 23) onto the PTC sample mounted on a copper cooler and placed into a basin made of a foam plastic. We used the lenses of 


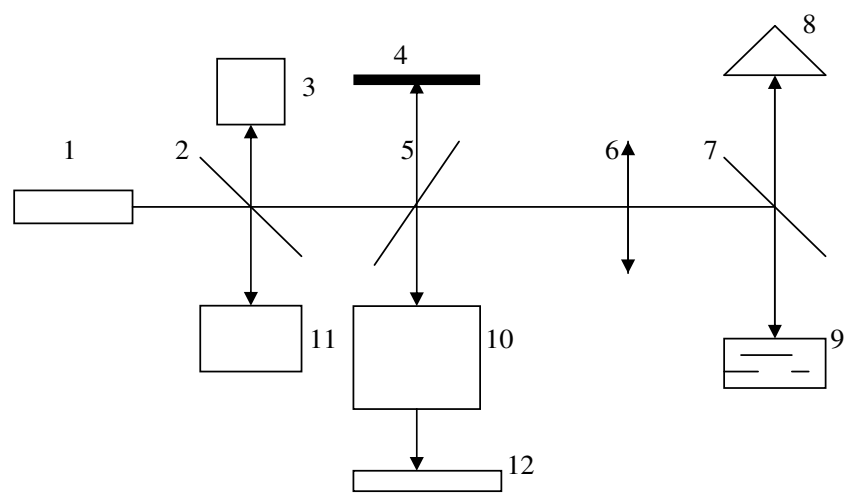

Fig. 23. The schematic of experimental setup for observing the Stimulated Globular Scattering (SGS) in the "backward" geometry; 1 - Ruby laser, 2, 5, 7 - half-transparent mirrors, 3, 8, 11 - power meters, 4 - the mirror, which can be removed (optional), 6 focusing system, 9 - the sample under study, 10 - the Fabri-Perot interferometer, 12 - minispectrometer.

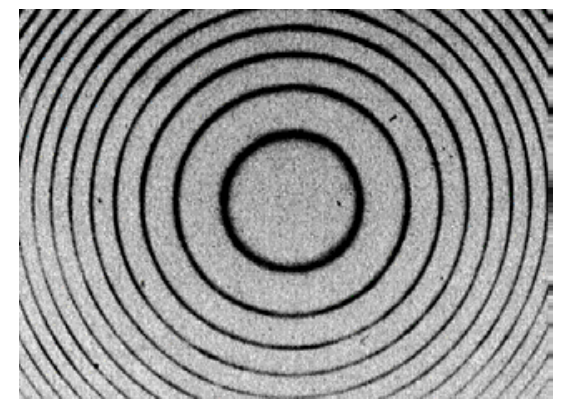

(a)

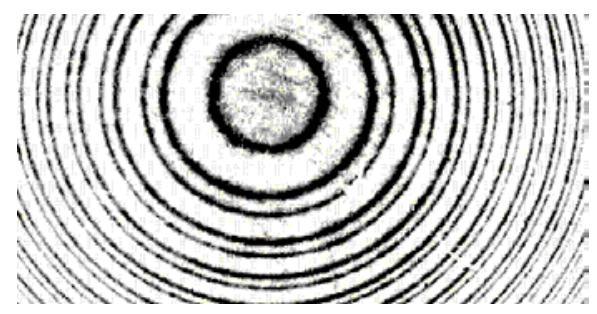

(b)

Fig. 24. The interferograms, obtained with the help of the Fabri-Perot interferometer, relating to the incident radiation spectrum of the Ruby laser $(\lambda=694.3 \mathrm{~nm})$, case (a), and to the spectrum of SGS in the "backward" geometry, case (b). In the second photo (case (b)) the system of double rings corresponds to the incident wave (the rings of smaller radius; the same rings can be found in the first photo, case (a)), and its scattering Stokes satellite (the rings of greater radius). In this particular case the free spectral range of the interferometer was equal to $0.833 \mathrm{~cm}^{-1}$. 
different focal lengths: 50, 90 and $150 \mathrm{~mm}$. Thus it was possible to perform the measurements for various intensities of the radiation as well as for various electromagnetic field distributions inside the sample. The PTC samples under study were manufactured of thin (their thickness was of $2-4 \mathrm{~mm}$ ) synthetic opal plates with the interface corresponding to the (111) crystal plane. The laser radiation was focused normally to the crystal interface. The scattered radiation in the "forward" geometry (Fig. 22) was analyzed in the incident wave direction with the help of the Fabri-Perot interferometer and a mini-spectrometer 7 . In case of the "backward" geometry (Fig. 23) the scattered radiation was analyzed in the opposite (with respect to the incident wave) direction with the help of the Fabri-Perot interferometer 10 and a mini-spectrometer 12. The mirror 4 (Fig. 23) was used for comparing the spectra of incident Ruby laser radiation and the scattered radiation.

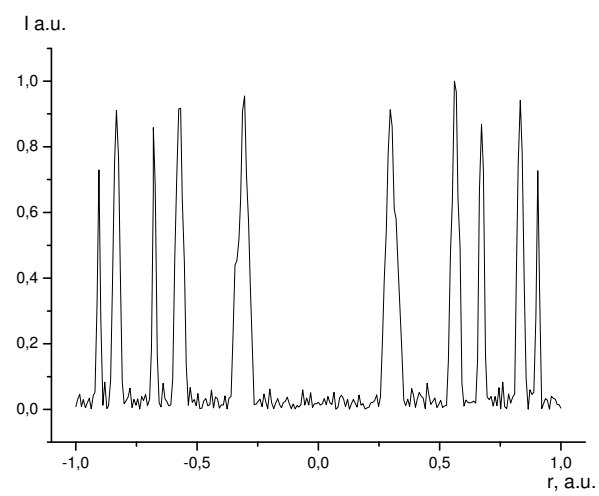

Fig. 25. The Stimulated Globular Scattering (SGS) spectrum in the PTC, obtained in the "backward" geometry. The free spectral range of the Fabri-Perot interferometer was of 0.833 $\mathrm{CM}^{-1}$. The broad lines are related to the incident laser radiation, while the narrow ones - to the Stokes satellites of the SGS.

The taking of the scattered radiation spectra was carried out at the room temperature, and also at cooling the samples up to the temperature of nitrogen boiling $(78 \mathrm{~K})$. In the latter case copper cooler with samples was placed into a foam plastic basin filled with the liquid nitrogen, while the crystal interface (111) was always above the level of the boiling nitrogen. For researching the spectra the Fabri-Perot interferometers with various (from 0.42 to 1.67 $\mathrm{cm}^{-1}$ ) free spectral ranges were used. The detectors 3 (Fig. 22), and 3, 8, 11 (Fig. 23) for measuring the energy of laser radiation and the signal of the SGS in the "forward" and "backward" geometry accordingly were applied.

In Fig. 24 (a) and (b) the interferograms related to the setup illustrated in Fig. 23 are given. Fig. 24 (a) shows the spectrum of incident Ruby laser radiation obtained at blocking the scattering signal by turning the half-transparent mirror 5 to the corresponding angle. In this case the spectral pattern had the form of the pattern of single interference rings, whose bandwidth was controlled by incident radiation bandwidth, which was on the level of 0.015 $\mathrm{cm}^{-1}$. Figs. 24 (b) and 25 are related to the experiment, where the scattered radiation was studied in the "backward" geometry. The patterns of double interference rings related to the incident radiation (the rings of smaller radius), and the Stokes satellites related to the SGS (the rings of greater radius) are clearly seen. The frequency shift in this case was about 0.44 
$\mathrm{cm}^{-1}$. The Stokes signal intensity appeared to be comparable with the incident radiation intensity. In the case of missing the mirror 4 (see Fig. 23), the spectrum would contain only the pattern of single rings caused by the SGS only, as in this case the incident radiation strongly diverges after its reflecting from the sample interface, and this signal does not input to the detector 12. We plotted the dependence of the frequency shift $f$ for the first Stokes component of the SGS as the function of the inverse diameter $(1 / D)$ of the globules. It occurred that such dependence is close to the linear one.

\section{f, $\mathrm{GHz}$}

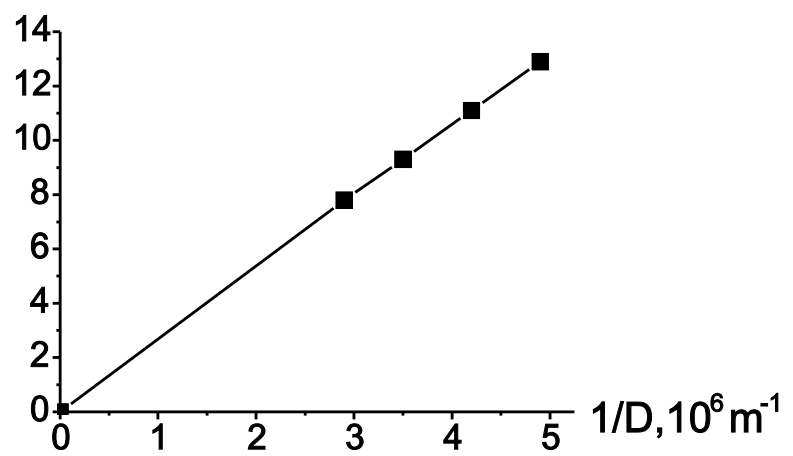

Fig. 26. Dependence of the frequency shift of frequency for the first Stokes component of the SGS versus the inverse diameter of the globules.

The explorations of spectra of the SGS were also performed for the opal matrices filled with liquids of various refractive index $n$ : water $(n=1.333)$, acetone $(n=1.359)$, ethanol $(n=$ $1.362)$, glycerol $(n=1.470)$, toluene $(n=1.497)$, benzene $(n=1.501)$ and nitrobenzene $(n=$ 1.553). Thus, the phase contrast (the value of $h=n / n S i O 2$, i.e. the ratio of refractive index of the liquid to that for quartz) changed in the range from 0.91 to 1.06. For example, impregnation of the opal matrix by acetone sharply decreases the phase contrast, and the sample becomes almost transparent. It provides an opportunity to observe the SGS in the scheme of the "forward" geometry of scattering. Similar to the case of the "backward" geometry, at the "forward" geometry of scattering the pattern of double rings related to the incident radiation (the rings of smaller diameter) and the Stokes component of the SGS (the rings of greater diameter) were seen as well. Due to the transparency of the sample treated by acetone it was possible to observe sufficiently intense Stokes signal of the scattering in the incident wave direction (the "forward" geometry). The Stokes shift in this case was on the level of $0.4 \mathrm{~cm}-1$. Note that the SGS was observed with the liquids pointed above both for the "forward" and "backward" geometry. In the case of "backward" geometry the frequency shift of about $0.4 \mathrm{~cm}-1$ was observed for the incident wave intensity at the level of $0.12 \mathrm{GW} / \mathrm{cm} 2$ for the opal matrices, filled with ethanol and acetone. The increase in the incident wave intensity up to $0.21 \mathrm{GW} / \mathrm{cm} 2$ resulted in occurrence of the second Stokes components having the frequency shift of about $0.65 \mathrm{~cm}-1$ for acetone, and $0.63 \mathrm{~cm}-1$ for ethanol. At the same time, for the "forward" geometry (and at the room temperature) only one Stokes component with the frequency shift of $0.4 \mathrm{~cm}-1$ was observed both for acetone, and for ethanol. 


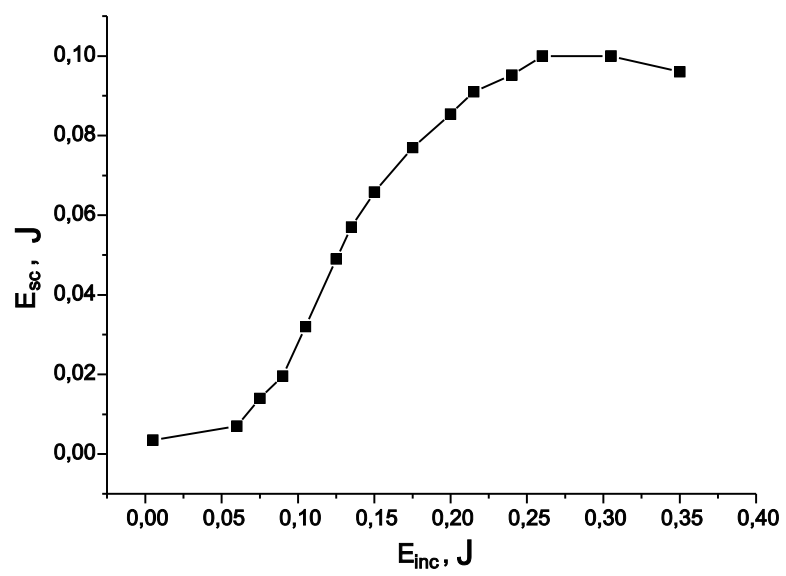

Fig. 27. Dependence of the energy $\left(\mathrm{E}_{\mathrm{sc}}\right)$ of the Stimulated Globular Scattering versus the energy of the incident wave $\mathrm{E}_{l}$ for the synthetic opals with the globule diameter of $245 \mathrm{~nm}$.

\begin{tabular}{|l|c|c|}
\hline $\begin{array}{l}\text { The geometry of } \\
\text { experiment }\end{array}$ & $\begin{array}{c}\text { The } \\
\text { frequency } \\
\text { shift, } \mathrm{cm}^{-1}\end{array}$ & $\begin{array}{c}\text { The number of } \\
\text { the Stokes } \\
\text { components }\end{array}$ \\
\hline $\begin{array}{l}\text { The "forward" } \\
\text { geometry, the opal } \\
\text { matrix is not } \\
\text { saturated by any } \\
\text { liquid }\end{array}$ & 0.44 & 1 \\
\hline $\begin{array}{l}\text { The "backward" } \\
\text { geometry, the opal } \\
\text { matrix is saturated by } \\
\text { acetone }\end{array}$ & 0.40 & 2 \\
\hline $\begin{array}{l}\text { The "forward" } \\
\text { geometry, the opal } \\
\text { matrix is saturated by } \\
\text { acetone }\end{array}$ & 0.65 & 1 \\
\hline $\begin{array}{l}\text { The "backward" } \\
\text { geometry, the opal } \\
\text { matrix is saturated by } \\
\text { ethanol }\end{array}$ & 0.40 & \\
\hline $\begin{array}{l}\text { The "forward" } \\
\text { geometry, the opal } \\
\text { matrix is saturated by } \\
\text { ethanol }\end{array}$ & 0.63 & 2 \\
\hline
\end{tabular}

Table 2. The values of the frequency shifts and the number of the Stokes components for various geometries of experiment. 
At the decrease of temperature up to $78 \mathrm{~K}$ the threshold for the SGS was three times reduced, and the number of observable Stokes components was increased. In the Table 2 the characteristic parameters of the Stokes components, including the frequency shift and the number of the Stokes component observed in the particular geometry of experiment are summarized.

As follows from this table, the values of the frequency shifts for the SGS appear close to those for the Stimulated Brillouin Scattering, observed earlier in the same liquid samples. The threshold for occurrence of SGS and the number of the Stokes components depend on the globule size, the substance in the crystal pores, the energy of incident laser radiation, and the temperature. In Fig. 27 the dependence of energy of the SGS versus the incident light energy is plotted for the liquid nitrogen temperature and for the globule diameter equal to $245 \mathrm{~nm}$ in case of the "backward" geometry. As is seen from the graph, at increasing the incident wave energy the SGS signal approaches the saturation level; note that the similar effect has been observed earlier for the Stimulated Raman Scattering. It is thus possible to explain the observed effect by increasing the efficiency of competing nonlinear processes at the growth of intensity of the incident radiation. The highest transformation factor of the incident radiation energy to the SGS energy obtained in our experiments was on the level of $60 \%$. As the quantum energy of the incident radiation is by four orders of magnitude higher than the energy of the corresponding radial vibrations of the globules, the intensity of acoustic phonons, generated by the globular vibrations should be approximately equal to $10^{3} \mathrm{~W} / \mathrm{cm}^{2}$. The lowest threshold for the SGS was realized for the so-called pulsating globular modes, the excitation of which should be accompanied by the oscillations of material density of the globules; this process is expected to have a high Qfactor. The corresponding acoustic waves have a scalar nature, since they are induced by radial vibrations of globules, and their propagation is not associated with any specific direction. In other words, these acoustic waves essentially differ from the acoustic waves of the vector type, i.e. from the transverse or longitudinal phonons. The wave equation for such scalar acoustic waves is analogous to the Klein - Gordon equation, describing the behavior of particles with the non-zero rest mass in the field theory:

$$
\left(\Delta-\frac{1}{S^{2}} \frac{\partial^{2}}{\partial t^{2}}\right) u(\mathbf{r}, t)=\frac{\omega_{0}^{2}}{S^{2}} u(\mathbf{r}, t),
$$

where $S$ has the sense of a group velocity of the wave at high values of the wave vector $\mathbf{k}$, $\omega_{0}=2 \pi c v_{0} \sim 1 / D, v_{0}$ is the corresponding magnitude of frequency of the pulsating mode, $u(\mathbf{r}, t)=u_{0} \exp (i \mathbf{k r}-\omega t)$ is the scalar wave function describing the propagation of a pulsating perturbation in space. According to (45), the dispersion law $\omega(k)$ for the scalar acoustic wave has the form:

$$
\omega^{2}=\omega_{0}^{2}+S^{2} k^{2}
$$

As was revealed in the experiments (see Fig. 26), the frequency shift of the first Stokes component of the SGS is inversely proportional to the globule diameter $D$, which is in conformity with Eqn. (46). For the "forward" geometry of experiment the wave vector of the scalar acoustic wave is equal to 


$$
k=k_{0}-k^{\prime},
$$

where $k_{0}$ and $k^{\prime}$ are wave vectors of the incident radiation and the SGS wave. If we deal with the "backward" geometry, we arrive at

$$
k=k_{0}+k^{\prime} .
$$

Thus, analyzing the spectra of the scattered radiation stimulated by the Ruby laser pulses in the PTC, we established that the spectral pattern is the set of double interference rings. These rings are related to the incident laser radiation and the Stokes components of the SGS. The intensity of the Stokes components is of the same order of magnitude as the incident wave intensity. This is an additional argument in favor of our statement, that the observed phenomenon is associated with the stimulated (not spontaneous) scattering.

\section{The conclusion}

Summarizing, we shown that implantation of various dielectrics, whose refractive index exceeds that for quartz, into the pores of synthetic opals results in shifting the band-gap of the PTC to lower frequencies. At the same time, at implantation of metals into these pores the band-gap is shifted to higher frequencies. Finally, implantation of various substances having the additional resonant absorption lines belonging to the band-gap of the crystal results in occurrence of additional sharp peaks of reflectance either in the long-wave or the short-wave areas of spectrum. The studies of characteristics of the PTC by the technique of reflectance spectroscopy of the band-gaps have revealed the areas of abnormal increase of density the photon states in the globular crystal; these areas are localized close to the band-gap boundaries. The analysis of such areas can allow us to obtain the lasing in the synthetic opals filled with active media. Besides, we expect that in the framework of this approach it will be possible to realize various nonlinear processes, including the Stimulated Raman Scattering of light, [9], Stimulated Globular Scattering of light $[6,9]$, generation of optical harmonics, parametrical generation [7] and the afterglow phenomena $[11,12]$.

On the basis of the results reported here we can make the following conclusions about the dynamics of acoustic phonons in the PTC.

1. The spectrum of acoustic phonons in the PTC contains the allowed and forbidden zones in the $\mathrm{GHz}$ frequency range, similar to the allowed and forbidden zones for the photons.

2. The group velocities of the acoustic phonons close to the forbidden zones (band-gaps) boundaries sharply decrease.

3. The effective mass of acoustic phonons close to the band-gap boundaries has an abnormally low magnitude, comparable to the mass of electron.

Let us also note that in the globular PTC a new type of standing acoustic elementary waves is possible [6,7]. These standing acoustic waves are induced in the globules and can be considered as the coupled states of pairs of the acoustic phonons - the so-termed biphonons. As was obtained in the experiments [6,7], such bi-phonons can be induced by the incident optical radiation, and the interaction between the optical wave and the bi-phonons leads to a new type of the stimulated light scattering - the SGS. 
Thus, the globular photonic crystal, being irradiated by powerful enough laser light can be generator of monochromatic acoustic waves in the $\mathrm{GHz}$ spectral range; the frequency of such waves should depend on the globule parameters and the type of a substance implanted into the crystal pores.

\section{Acknowledgement}

The given work was supported by the Russian Foundation for Basic Researches, Grants Nos. 08-02-00114, 10-02-00293, 10-02-90042-Bel, 10-02-90404-Ukr, and by the Presidium of Russian Academy of Sciences, Program for Basic Researches No. 21.

\section{References}

[1] Yablonovich, E. (1987). Inhibited Spontaneous Emission in Solid-State Physics and Electronics, Physical Review Letters, Vol. 58, No. 20, pp. 2059 - 2062.

[2] John, S. (1987). Strong Localization of Photons in Certain Disordered Dielectric Superlattices, Physical Review Letters, Vol. 58, No. 23, pp. 2486-2489.

[3] Gorelik, V.S., Zlobina, L.I., Troitskii, O.A., et al. (2008). LED-excited emission of opal loaded with silver nanoparticles, Inorganic Materials, Vol. 44, No. 1, pp. 58 61.

[4] Goncharov, A.P., Gorelik, V.S. (2007). Emission of opal photonic crystals under pulsed laser excitation, Inorganic Materials, Vol. 43, No. 4, pp. 386 - 391.

[5] Bunkin, N.F., Gorelik, V.S., Filatov, V.V. (2010). Acoustic properties of globular photonic crystals based on synthetic opals, Physics of Wave Phenomena, Vol. 18, No. 2, pp. 90 95.

[6] Gorelik, V.S. (2007). Optics of globular photonic crystals, Quantum Electronics, Vol. 37, No. 5, pp. 409-432.

[7] Gorelik, V.S. (2008). Optics of globular photonic crystals, Laser Physics, Vol. 18, No. 12, pp. 1479-1500.

[8] Voshchinskii, Yu.A., Gorelik, V.S. (2011). Dispersion law in photonic crystals in sinusoidal and quasi-relativistic approximation, Inorganic Materials, Vol. 47, No. 2, pp. $148-151$.

[9] Gorelik, V.S. (2010). Linear and nonlinear optical phenomena in nanostructured photonic crystals, filled by dielectrics or metals, European Journal - Applied Physics, Vol. 49, No. 3, 3307.

[10] Kuok, M.H., Lim, H.S., Ng, S.C., Liu, N.N., Wang, Z.K. (2003). Brillouin study of the quantization of acoustic modes in nanospheres, Physical Review Letters, Vol. 90, No. 25, 255502.

[11] Gorelik, V.S., Kudryavtseva, A.D., Tareeva, M.V., et al. (2006). Spectral characteristics of the radiation of artificial opal crystals in the presence of the photonic flame effect, JETP Letters, Vol. 84, No. 9, pp. 485-488.

[12] Gorelik, V.S., Esakov, A.A., Zasavitskii, I.I., (2010), Low-temperature persistent afterglow in opal photonic crystals under pulsed UV excitation, Inorganic Materials, Vol. 46, No. 6, pp. 639-643. 
[13] Gorelik, V.S., Yurasov, N.I., Gryaznov, V.V., et al., (2009), Optical properties of threedimensional magnetic opal photonic crystals, Inorganic Materials, Vol. 45, No. 9, pp. 1013-1017. 


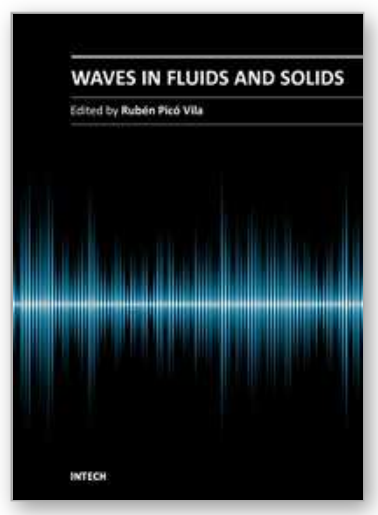

\author{
Waves in Fluids and Solids \\ Edited by Prof. Ruben Pico Vila
}

ISBN 978-953-307-285-2

Hard cover, 314 pages

Publisher InTech

Published online 22, September, 2011

Published in print edition September, 2011

Acoustics is an discipline that deals with many types of fields wave phenomena. Originally the field of Acoustics was consecrated to the sound, that is, the study of small pressure waves in air detected by the human ear. The scope of this field of physics has been extended to higher and lower frequencies and to higher intensity levels. Moreover, structural vibrations are also included in acoustics as a wave phenomena produced by elastic waves. This book is focused on acoustic waves in fluid media and elastic perturbations in heterogeneous media. Many different systems are analyzed in this book like layered media, solitons, piezoelectric substrates, crystalline systems, granular materials, interface waves, phononic crystals, acoustic levitation and soft media. Numerical methods are also presented as a fourth-order Runge-Kutta method and an inverse scattering method.

\title{
How to reference
}

In order to correctly reference this scholarly work, feel free to copy and paste the following:

N. F. Bunkin and V. S. Gorelik (2011). Acoustic Properties of the Globular Photonic Crystals, Waves in Fluids and Solids, Prof. Ruben Pico Vila (Ed.), ISBN: 978-953-307-285-2, InTech, Available from: http://www.intechopen.com/books/waves-in-fluids-and-solids/acoustic-properties-of-the-globular-photoniccrystals

\section{INTECH}

open science | open minds

\author{
InTech Europe \\ University Campus STeP Ri \\ Slavka Krautzeka 83/A \\ 51000 Rijeka, Croatia \\ Phone: +385 (51) 770447 \\ Fax: +385 (51) 686166 \\ www.intechopen.com
}

\author{
InTech China \\ Unit 405, Office Block, Hotel Equatorial Shanghai \\ No.65, Yan An Road (West), Shanghai, 200040, China \\ 中国上海市延安西路65号上海国际贵都大饭店办公楼 405 单元 \\ Phone: +86-21-62489820 \\ Fax: +86-21-62489821
}


(C) 2011 The Author(s). Licensee IntechOpen. This chapter is distributed under the terms of the Creative Commons Attribution-NonCommercialShareAlike-3.0 License, which permits use, distribution and reproduction for non-commercial purposes, provided the original is properly cited and derivative works building on this content are distributed under the same license. 